\title{
Oscillatory fluid motion unlocks plug flow operation in helical tube reactors at lower Reynolds numbers $(\operatorname{Re} \leq 10)$
}

\author{
J.R. McDonough ${ }^{\mathrm{a}}$, S. Murta ${ }^{\mathrm{a}}$, R. Law ${ }^{\mathrm{a}}$, A.P. Harvey \\ ${ }^{a}$ School of Engineering, Merz Court, Newcastle University, Newcastle upon Tyne, NE1 7RU, UK \\ ${ }^{\dagger}$ Corresponding Author
}

\begin{abstract}
Flow in helical tubes exhibits plug flow characteristics above a critical flow rate due to the formation of Dean vortices. In this study, we report the novel use of oscillatory flow inside coiled tubes to achieve high degrees of plug flow at lower flow rates. The plug flow enhancement appeared to 'switch-on' then 'switch-off' as the oscillation intensity was increased, corresponding to 'oscillatory Dean numbers' of $\mathrm{De}_{\mathrm{o}}=70-200$, where secondary instabilities are expected. It is believed that oscillatory motion leads to the periodic formation and unravelling of Dean vortices. For a $5 \mathrm{~mm}$ tube diameter, the effects of radius of curvature $(12.5-32.5 \mathrm{~mm})$, tube pitch $(7.5-12.5 \mathrm{~mm})$, net flow rate $\left(\operatorname{Re}_{\mathrm{n}}=10-50\right)$, oscillation frequency $(2-8 \mathrm{~Hz})$ and oscillation amplitude $(1-8 \mathrm{~mm})$ were studied. The prototype helical coil reactors were rapidly manufactured using 3D printing. The optimal conditions for plug flow corresponded to $\mathrm{De}_{\mathrm{o}} / \mathrm{Re}_{\mathrm{n}}=2-8$, at Strouhal numbers in the range $\mathrm{St}=1-2$. Plug flow was observed at net flow Reynolds numbers at least as low as $\operatorname{Re}_{\mathrm{n}}=10$, compared to $\operatorname{Re}_{\mathrm{n}}=70-300$ in the literature for coiled tubes subjected to steady flows.
\end{abstract}

Keywords: Plug flow, residence time distribution, Dean vortex, oscillation, helical tube reactor

\section{Graphical Abstract:}

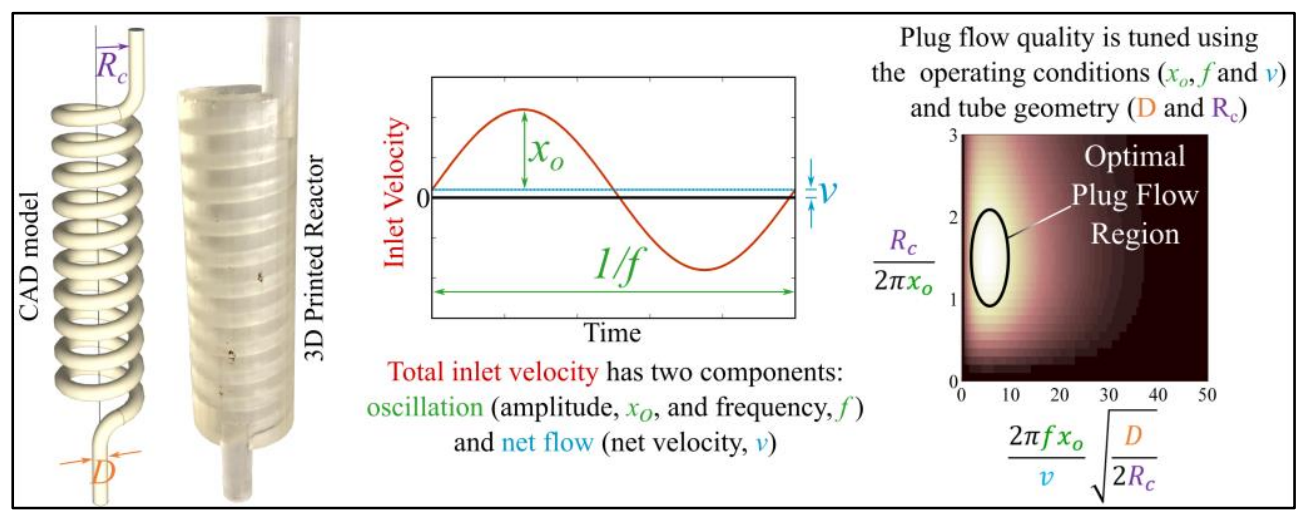




\section{Introduction}

Liquids flowing in coiled tubes experience centrifugal forces that shift the maximum point of the radial velocity distribution towards the outer side of the curve. This increases the pressure at the outside wall (further from the centre of curvature) relative to the inside wall (closer to the centre of curvature), producing a pressure gradient that acts across the tube cross-section that balances the centrifugal force [1]. This is known as the Dean instability, first described by Dean [2]. Because the liquid at the top and bottom of the tube moves slower than the liquid at the tube's centre (an effect of the no-slip boundary condition), there is less centrifugal force in this peripheral region, requiring a smaller pressure gradient to balance the outward motion [1]. Therefore, beyond a critical liquid velocity, the pressure gradient will dominant the centrifugal force at the tube wall, resulting in a flow of liquid at the top and bottom of the tube towards the inner wall, with a restoring flow at the centre of the tube towards the outer wall. This secondary flow manifests as a pair of counter-rotating vortices (Figure 1a), known as Dean vortices, which are superimposed onto the net motion. The dimensionless Dean number (equation 1) can be used to predict the onset of these secondary flows [3]. The Dean number contains terms accounting for the fluid's inertia and internal viscous dissipation, and centripetal forces induced as the fluid's direction of motion changes. Dual vortex pairs are observed for De > 64-75, whilst additional instabilities (such as vortex splitting/merging and undulations) are observed for De $=75-200$ [4].

$$
D e=\sqrt{\frac{\frac{1}{2} \text { (inertial forces)(centripetal forces) }}{\text { viscous forces }}}=\operatorname{Re} \sqrt{\frac{D}{2 R_{c}}}
$$

In equation $1, \mathrm{Re}$ is the Reynolds number (shown in equation 2 ) that defines the ratio of inertial to viscous forces, where $\rho$ is the fluid density, $\mathrm{v}$ is the net flow velocity, $\mathrm{D}$ is the tube diameter and $\mu$ is the fluid viscosity. It can be inferred from equation 1 that viscous forces dissipate the inertial and centripetal forces, i.e. higher viscosity fluids require larger tube diameters (D), smaller radii of curvature $\left(\mathrm{R}_{\mathrm{c}}\right)$ and higher fluid velocities to induce the Dean instability.

$$
R e=\frac{\rho v D}{\mu}
$$
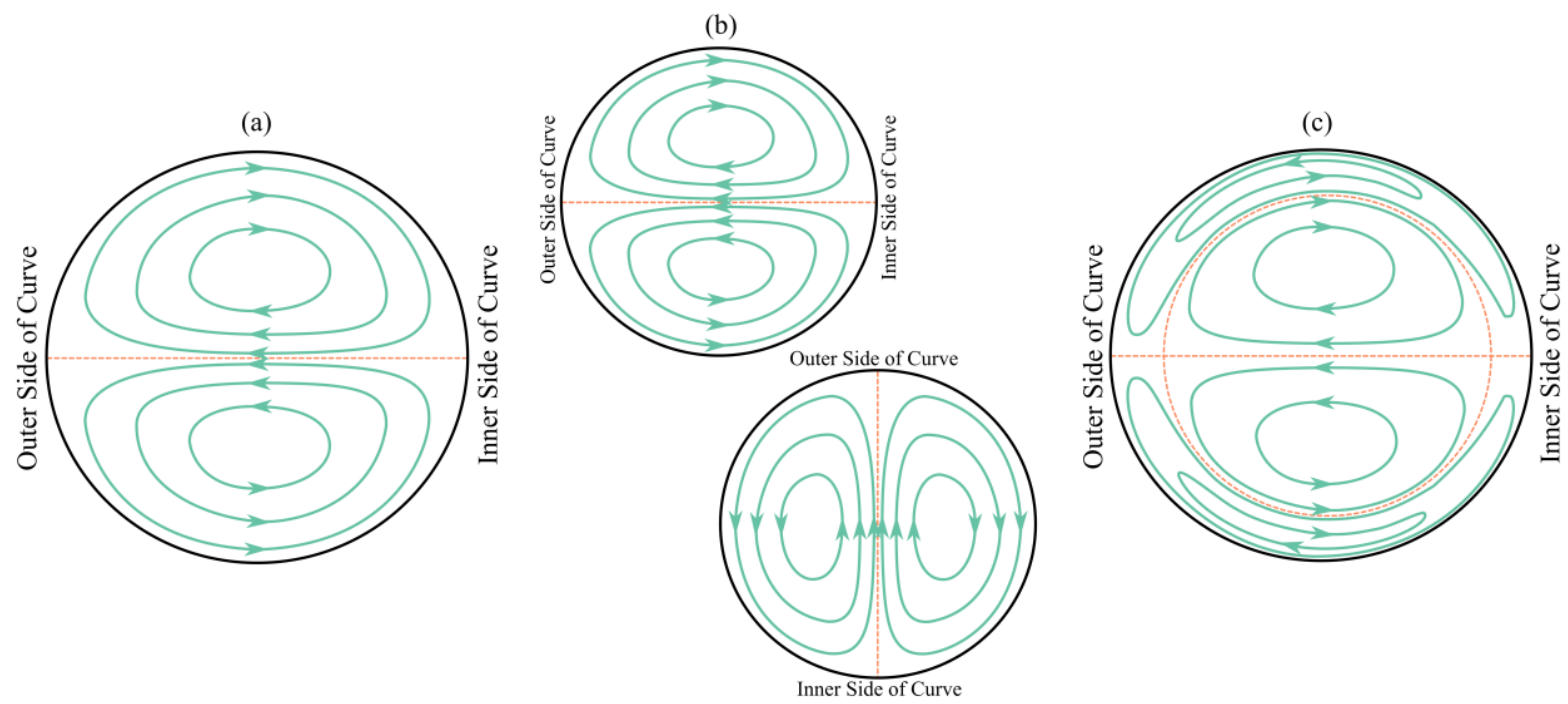

Figure 1. (a) Schematic of a pair of Dean vortices observed in the cross-section of a coiled tube, (b) rotation of Dean vortices when the direction of curvature changes in coiled flow inverters [5], and (c) the Lyne instability observed in pulsatile helical coil flows [6]

Plug flow is a desirable operating condition characterised by strong cross-channel mixing and negligible axial mixing. Using the Dean vortex phenomenon, multiple studies have shown that coiled tubes reduce axial dispersion compared to straight tubes in the laminar regime $[5,7,8,9]$. Here, greater frictional losses at the outer side of the curve are converted into improved radial mixing. However, it has been shown previously that coiled tubes only provide an advantage over straight tubes (where lower axial 
dispersion is produced) for Reynolds numbers of $\mathrm{Re}>300$ [7]. This provides a lower limit for the flow rates. For long residence time processes, long reactors would be required that might be impractical depending on the application. Further, the fluid at the centre of the Dean vortices can only "escape" via diffusion [10]; the presence of these fluid trapping regions means the mixing and axial dispersion performance are sub-optimal.

A number of approaches have consequently been investigated to further improve the mixing and plug flow characteristics of coiled tube reactors. A commonly reported approach is the coiled flow inverter, with numerous applications emerging for protein refolding [11], polymerisation [12], extraction [13] and biotherapeutics [14]. Coiled flow inverters (CFIs) are helical coils containing periodic bends that change the direction of centrifugal force in the tube. This causes the plane in which Dean vortices form to also rotate by the same angle [5]. Thus, by periodically bending the coils by $90^{\circ}$ bends (Figure 2), the Dean vortices will also be periodically rotated by $90^{\circ}$ (Figure $1 \mathrm{~b}$ ). These inversions promote better radial mixing, helping to lessen the impact of the stagnation points at the centres of the Dean vortices.

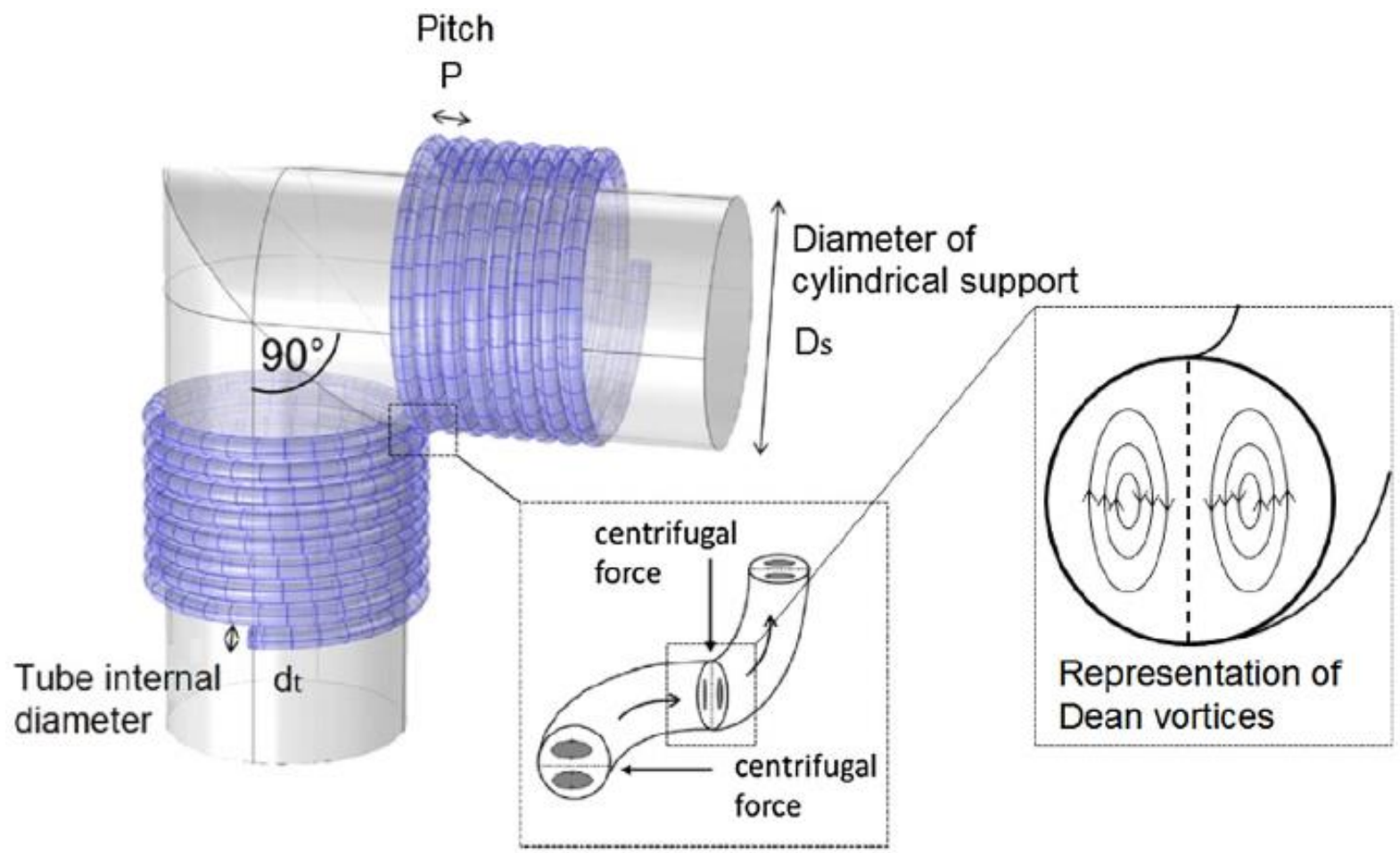

Figure 2. Coiled Flow Inverter (CFI) with a single $90^{\circ}$ inversion [15]

Coiled flow inverters have also been proposed as a method for enhancing heat transfer rates. For example, Singh \& Nigam [16] recently reported the pilot scale results of three CFI heat exchangers, where the entire coil assemblies (stainless steel) were placed inside a shell. Here, different banks of CFIs were connected in series, with each bank comprising $490^{\circ}$ bends with 4 turns of the coil between each bend. They observed a factor of 2.2-4.5 enhancement of the overall heat transfer coefficient compared to a conventional shell-and-tube heat exchanger using a surface area that was a factor of 8 smaller, representing a total intensification factor of 4.4-36. However, the packing (space) efficiency of these coils in the shell was not presented. It would also be conceivably difficult to efficiently rapidly adjust the temperature in a CFI because of the large thermal mass required on the shell side; typical labscale setups thus far have involved submerging the entire tube assembly in a heat transfer medium [17].

Helically coiled tubes also improve the heat transfer rates compared to conventional straight tubes [18], and they are generally simpler to fabricate than CFIs. Because coiled tubes are already established in many industrial applications [18], a 'retrofittable' solution for augmenting the heat transfer rate, and other performance characteristics, in existing helical coil tubes would be preferred. 
Pulsatile/oscillatory flows have also been investigated as an alternative means for improving the mixing response in helical coil reactors, which may provide the solution for augmenting the performance of an existing helical coil reactor with minimal process disturbance. For instance, Jarrahi et al. [19] reported detailed PIV results for a single $90^{\circ}$ bend under laminar conditions $(\mathrm{Re}=420-1000, \mathrm{De}=126.6-301.5)$ subject to oscillatory flows with ratios of maximum oscillatory velocity to steady velocities of $\beta=1-4$ and dimensionless oscillation frequencies of $\alpha=R_{c}(\omega / v)^{0.5}=8.37-24.5$. They found that even for 'unfavourable' oscillatory conditions, the time-averaged vorticity and shear strain rates were still 5$15 \%$ higher than those obtained under steady flow conditions. They also reported up to $400 \%$ improvements in the vorticity and shear for $\beta \geq 2$. Jarrahi et al. [19] observed numerous complex timedependent structures, including no-vortices, two-vortices (ordinary Dean flow), four-vortices and single-vortices for $\beta \geq 1$; similar to those previously observed by Sudo et al. [20]. Timité et al. [21] similarly describe the appearance of more complex structures such as Lyne instabilities (Figure 1c) and swirling structures. They postulate that the oscillatory motion results in the continuous destruction and reformation of the vortices that reduces the influence of the stagnant 'islands' at the centres of the vortices. Jarrahi et al. [22] later confirmed that the four-vortex condition resulted in improved radial flow in the channel cross-section compared with the two-vortex condition produced in steady flows.

It has been demonstrated in numerous studies that the oscillatory motion in combination with tube inserts also improves the mixing characteristics in straight tubes. Intensification has subsequently been demonstrated for heat exchange [23], fermentation [24], crystallization [25], biodiesel and biofuels production (e.g. [26]), water treatment using ozone [27], suspension polymerisation [28] and biolubricant synthesis [29]. These 'oscillatory baffled reactors' (OBRs) have also been scaled down to $\mathrm{mL} / \mathrm{hr}$ throughputs for process development and reaction screening applications [30]. The oscillatory motion in these OBRs decouples the mixing intensity from the net flow rate, allowing substantially shorter length to diameter ratios to achieve a given mean residence time. The oscillatory motion in combination with the tube inserts also has a strong positive influence on the residence time distribution (RTD) performance $[31,32]$, providing precedent for the use of oscillatory motion in helical coils for plug flow generation/control, which has not yet been investigated.

Consequently, the aim of this study is to ascertain whether combining coiled tube geometries with fully oscillatory motion (opposed to pulsatile flow) can enhance the plug flow performance. Through analogy with the OBR, the expected novelty is plug flow enhancement at flow rates lower than previously reported, potentially opening up new applications, or reduced reactor size for a given throughput. Oscillatory motion is also easy to implement, so may provide a 'retrofittable' solution for enhancing existing processes that use helical coils. In this study the plug flow will be assessed by measuring the RTD in different coil geometries (with different radii of curvature and tube pitches) subject to a range of net flow rates and oscillation intensities. The present study is limited to a single tube diameter in order to simplify the dataset whilst providing a proof-of-concept for the idea. The data in this study will also be used to compare the plug flow performance of the helical tubes subject to oscillatory flows with the similar oscillatory baffled reactor and coiled flow inverter. 


\section{Methodology}

\subsection{Helical Coil Reactor Designs}

A schematic of the helical coil geometries is shown in Figure 3a, whilst the characteristic dimensions of each coil used in the study are summarised in Table 1. Figures $3 \mathrm{~b}$ and $3 \mathrm{c}$ also respectively show a $\mathrm{CAD}$ drawing and image of the final 3D printed product of reactor geometry ' $\mathrm{R} 1$ '. The hole included at the centre was to reduce the amount of resin required for the build.

Table 1. Summary of helical coil geometries

\begin{tabular}{llllllll}
\hline \multirow{3}{*}{ Reactor } & $\begin{array}{l}\text { Tube } \\
\text { Diameter, } \\
\mathrm{D}_{\mathrm{t}}(\mathrm{mm})\end{array}$ & $\begin{array}{l}\text { Radius of } \\
\text { Curvature, } \\
\mathrm{R}_{\mathrm{c}}(\mathrm{mm})\end{array}$ & $\begin{array}{l}\text { Tube } \\
\text { Pitch, } \mathrm{P} \\
(\mathrm{mm})\end{array}$ & $\begin{array}{l}\text { Number } \\
\text { of Turns, } \\
\mathrm{T}\end{array}$ & $\begin{array}{l}\text { Reactor } \\
\text { Volume, } \\
\mathrm{V}(\mathrm{mL})\end{array}$ & $\begin{array}{l}\text { Coil } \\
\text { Length, } \mathrm{L}_{\mathrm{c}} \\
(\mathrm{mm})\end{array}$ & $\begin{array}{l}\text { Total } \\
\text { Length, } \\
\mathrm{L}_{\mathrm{T}}(\mathrm{mm})\end{array}$ \\
\hline $\mathrm{R} 1$ & 5 & 12.5 & 10.0 & 10 & 16.48 & 948 & 1008 \\
$\mathrm{R} 2$ & 5 & 22.5 & 10.0 & 6 & 17.19 & 944 & 1005 \\
$\mathrm{R} 3$ & 5 & 32.5 & 10.0 & 4.25 & 17.55 & 936 & 996 \\
$\mathrm{R} 4$ & 5 & 12.5 & 7.5 & 10 & 16.00 & 945 & 1006 \\
$\mathrm{R} 5$ & 5 & 12.5 & 12.5 & 10 & 16.12 & 951 & 1011 \\
\hline
\end{tabular}

(a)

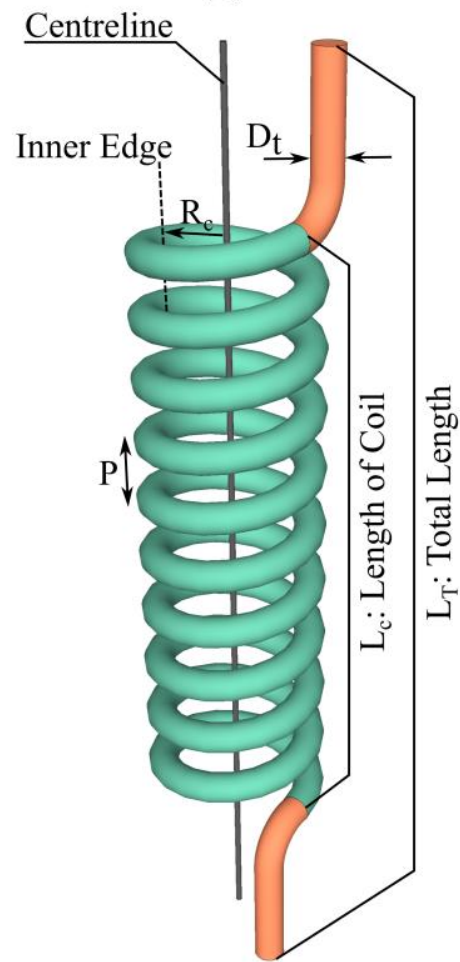

(b)

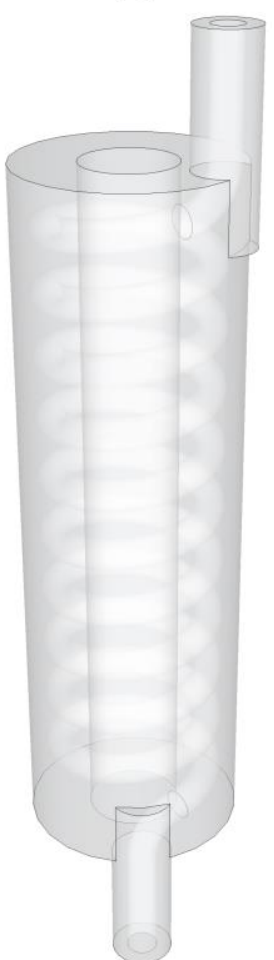

(c)

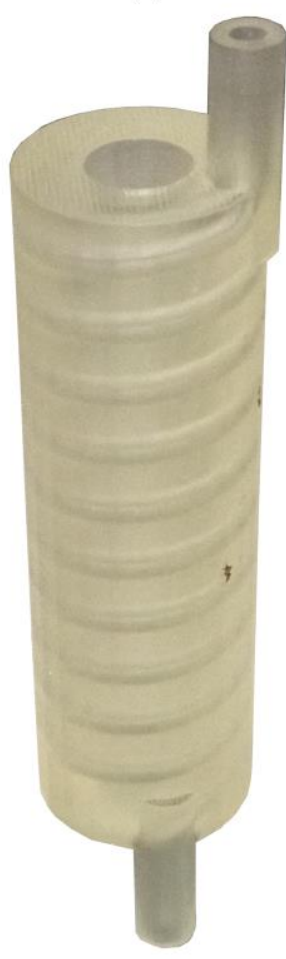

Figure 3. (a) Schematic of helical coil geometric parameters, (b) example CAD drawing of a helical coil reactor (design RI), and (c) example of final $3 D$ printed reactor (design $R 1$ )

All designs in the present study used a $5 \mathrm{~mm}$ tube diameter, based on the mesoscale oscillatory baffled reactor (meso-OBR) [30]. This provided a set of benchmark data to assist in the understanding of the results. Additionally, one of the main design advantages of meso-OBRs compared to microchannel reactors is their ability to suspend solid particles 'off the shelf' and accommodate multi-phase processes with minimal optimisation of the contacting strategy. This means the results of the present study would retain this design advantage when applied to flow chemistry applications.

The volumes of the different coil designs were held approximately constant, around 16-17 $\mathrm{mL}$, by changing the number of turns of the coil depending on the radius of curvature. This removed the mean residence time effect from the tracer injection experiments, allowing for direct comparison of the effects of: tube pitch, radius of curvature, and oscillation frequency and amplitude. Short straight sections of 
tubing were also placed at the entrance and exit of the reactor coil (highlighted in orange in Figure 5a) so the reactors could be connected to tube fittings that housed the conductivity probe and linked the reactors with the syringe pumps.

\subsection{Fabrication of the Reactors Using Additive Manufacturing}

Each of the reactor geometries in Table 1 was designed using Google SketchUp, with the helical path of the coil defined using the "Curve Maker" plug-in, and 3D printed using a Form2 (FormLabs) printer. The Form2 uses stereolithography (SLA) technology, which fabricates objects layer by layer via photopolymerisation of a resin. In this study, the clear resin formulation was used, which contains a mix of methyl acrylate monomers/oligomers and photoinitiators, because this allowed the presence of any air bubbles that might have hindered the fluid mechanics to be identified and subsequently purged. Each layer during the print was created using a $405 \mathrm{~nm}$ UV laser $(140 \mu \mathrm{m}$ spot size, $250 \mathrm{~mW})$ that quickly scanned through the tank to define the profile of the particular slice through the geometry. Then, the build platform would move upwards allowing the next layer to be created on the previous layer. The maximum XY (horizontal) resolution was $150 \mu \mathrm{m}$, and all parts were printed with the $100 \mu \mathrm{m}$ layer height setting because this minimised the build time and did not provide any noticeable improvement in surface quality compared with the $25 \mu \mathrm{m}$ layer height setting. Following printing, the parts were washed in IPA using an ultrasonic wash bath (FormWash), then dried with compressed air before being post-cured at $60^{\circ} \mathrm{C}$ for $90 \mathrm{~min}$ using $405 \mathrm{~nm}$ UV light (using a FormCure).

Due to the layering process and finite resolution of the 3D printer, a "stair stepping effect" typically occurs for inclined surfaces. This effect is particularly severe for near-horizontal angles and low layer height resolutions. Further, the wall roughness on surfaces aligned with the printing direction can still be significant (of the order of $\mu \mathrm{m}$ ) unless polished. MacDonald et al. [33] compared three different 3D printing platforms for the fabrication of microfluidic devices: Fused Deposition Modelling (FDM), Polyjet and Digital Light Processing Stereolithography (DLP-SLA). The resolution of their DLP-SLA printer was $56 \times 56 \times 50 \mu \mathrm{m}$, producing a final surface roughness of $0.35 \mu \mathrm{m}$, and relative roughness (roughness divided by channel width) of 0.00047 . In laminar flow tests (at flow rates of $25-100 \mu \mathrm{L} / \mathrm{min}$ ) in a $750 \times 500 \mu \mathrm{m}$ channel, MacDonald et al. [33] found that the extent of mixing was significantly lower in the DLP-SLA device compared to the FDM technology, which had a surface roughness of $10.97 \mu \mathrm{m}$. Further, the flow profiles in the DLP-SLA microfluidic device were comparable to more conventional fabricated materials in the laminar regime. In the Form2 printer used in this study, the root mean square surface roughness was $2.9 \mu \mathrm{m}$ (using the $100 \mu \mathrm{m}$ layer height setting) [34]. Thus, the relative roughness of the helically coiled channels in this study was 0.00058 , similar to the value reported by MacDonald et al. [33]. Therefore, it is likely that the bulk micro-mixing performance was not substantially affected by the surface roughness. Further, Okafor et al. [34] successfully applied the Form2 printer for the fabrication of a $2.5 \mathrm{~mm}$ diameter meso-OBR, reporting Gaussian RTDs that indicated vortex formation was minimally influenced by the surface roughness.

\subsection{Operating Conditions}

The plug flow behaviour was assessed across a wide range of net flow rates and oscillation amplitudes and frequencies. In the similar meso-OBR platform, the net flow rate is characterised using the net flow Reynolds number, $\operatorname{Re}_{\mathrm{n}}$, (equation 2) whilst the oscillation intensity is characterised using the oscillatory Reynolds number (equation 3) [30]. Here, $\rho$ is the fluid density, D is the tube diameter, $\mu$ is the fluid viscosity, $\mathrm{f}$ is the oscillation frequency and $\mathrm{x}_{\mathrm{o}}$ is the oscillation amplitude. In equation 3 , the net flow velocity " $\mathrm{v}$ " is replaced with the maximum oscillatory velocity " $2 \pi \mathrm{f}_{\mathrm{o}}$ ". Both of these groups define the ratio of inertial to viscous forces; in the case of $\mathrm{Re}_{\mathrm{n}}$, the inertia is described according to the net flow rate whilst for $\mathrm{Re}_{\mathrm{o}}$, the inertia is described by the oscillation intensity. Because these equations are usually applied for reactors operating in the laminar regime, it is $\mathrm{Re}_{\mathrm{o}}$ that mainly characterises the amount of "turbulence", in the form of a "laminar unsteadiness" [35].

$$
R e_{o}=\frac{2 \pi f x_{o} \rho D}{\mu}
$$

Helical coils were the focus of this study, which are usually characterised by the Dean number (equation 1). This takes into consideration the effects of inertial, centripetal and viscous forces, and defines the 
onset of secondary flows in the form of Dean vortices via the pressure and velocity gradients in the coiled tube [3]. Consequently, analogously to the oscillatory Reynolds number, an 'oscillatory Dean number' (equation 4) was proposed for this study, where the oscillatory velocity component accounted for the fluid's inertia.

$$
D e_{o}=R e_{o} \sqrt{\frac{D}{2 R_{C}}}
$$

Net flow Reynolds numbers of $\mathrm{Re}_{\mathrm{n}}=10-50$, corresponding to mean residence times of 90-500 s (based on the volumes in Table 1), were investigated in each of the five reactor geometries, covering a reasonable range of conditions that are applicable for flow chemistry applications. Oscillation frequencies of $1-8 \mathrm{~Hz}$ and oscillation amplitudes of $1-8 \mathrm{~mm}$ were simultaneously applied, although the combined effects of high amplitude and high frequency could not be established because of a limit of the syringe pump. Table 2 summarises the combinations of frequencies and amplitudes that could be tested. Nevertheless, these conditions are comparable to those widely applied in the similar meso-OBR platform [30], and as demonstrated in this paper, the optimum conditions for each reactor geometry existed within the range studied, showing there is minimal scope for studying oscillatory conditions outside of this range. Additionally, a set of control experiments were performed with no oscillatory motion to assess the impact of the oscillatory motion on the plug flow response. Each operating condition was performed in triplicate. The experimental errors were the standard deviations of the data observed across these three experiments.

Table 2. Oscillation condition matrix showing obtainable (green) and unobtainable (red) combinations of oscillation amplitude $\left(x_{o}\right)$ and frequency $(f)$ with the syringe pump

\begin{tabular}{|c|c|c|c|c|c|}
\hline \multirow{2}{*}{$\mathrm{f}(\mathrm{Hz})$} & \multicolumn{5}{|c|}{$\mathrm{x}_{\mathrm{o}}(\mathrm{mm})$} \\
\hline & 1 & 2 & 3 & 4 & 8 \\
\hline 2 & & & & & \\
\hline 3 & & & & & \\
\hline 4 & & & & & \\
\hline 5 & & & & & \\
\hline 6 & & & & & \\
\hline 7 & & & & & \\
\hline 8 & & & & & \\
\hline
\end{tabular}

\subsection{Tracer Injection}

\subsubsection{Experiment Configuration and Procedure}

The plug flow behaviour was assessed using residence time distributions (RTDs) that were experimentally measured using standard tracer pulse experiments. Here, deionised water was used to generate the main flow, and a $0.5 \mathrm{M} \mathrm{KCl}$ solution was used as the tracer. Based on the mass of $\mathrm{KCl}$ dissolved, this represents a density increase compared to deionised water of just $37 \mathrm{~kg} / \mathrm{m}^{3}$. Phan et al. [36] subsequently demonstrated that tracer concentrations in the range of 0.3-3 $\mathrm{M}$ have no effect on the shape of the dimensionless RTD curve in vertical oscillatory flows in the presence of tube inserts. Thus, based on the rise angle of coil and enhanced radial mixing via the formation of Dean vortices, it can be assumed that the tracer density had negligible impact on the measured RTDs in this study.

Figure 4 shows an image of the experiment setup. A Swagelok Tee-junction with $10 \mathrm{~mm}$ tube fittings on the through-section and a $4 \mathrm{~mm}$ tube fitting on the branch was connected to the outlet section of the coiled reactors. A conductivity probe (CDC749, Hach) could then be inserted into the flow through the branched section of this fitting to measure the conductivity of the salt tracer at the exit of the coil. Deadzones inside the fitting were eliminated by fitting a custom 3D printed plug around the probe tip, meaning the interior of each Swagelok fitting consisted of just a $5 \mathrm{~mm}$ diameter cylinder (highlighted in Figure 4). The conductivity probe was then connected to a CDM210 conductivity meter, with the analogue output routed to an ADC-20 data logger that was monitored using PicoLog software. An extended run of tube, with a disengagement zone at the top, was fitted to the upper tee-junction in order 
to prevent the accumulation of tracer around the outlet conductivity probe that, through experience, has been found to skew the RTD.

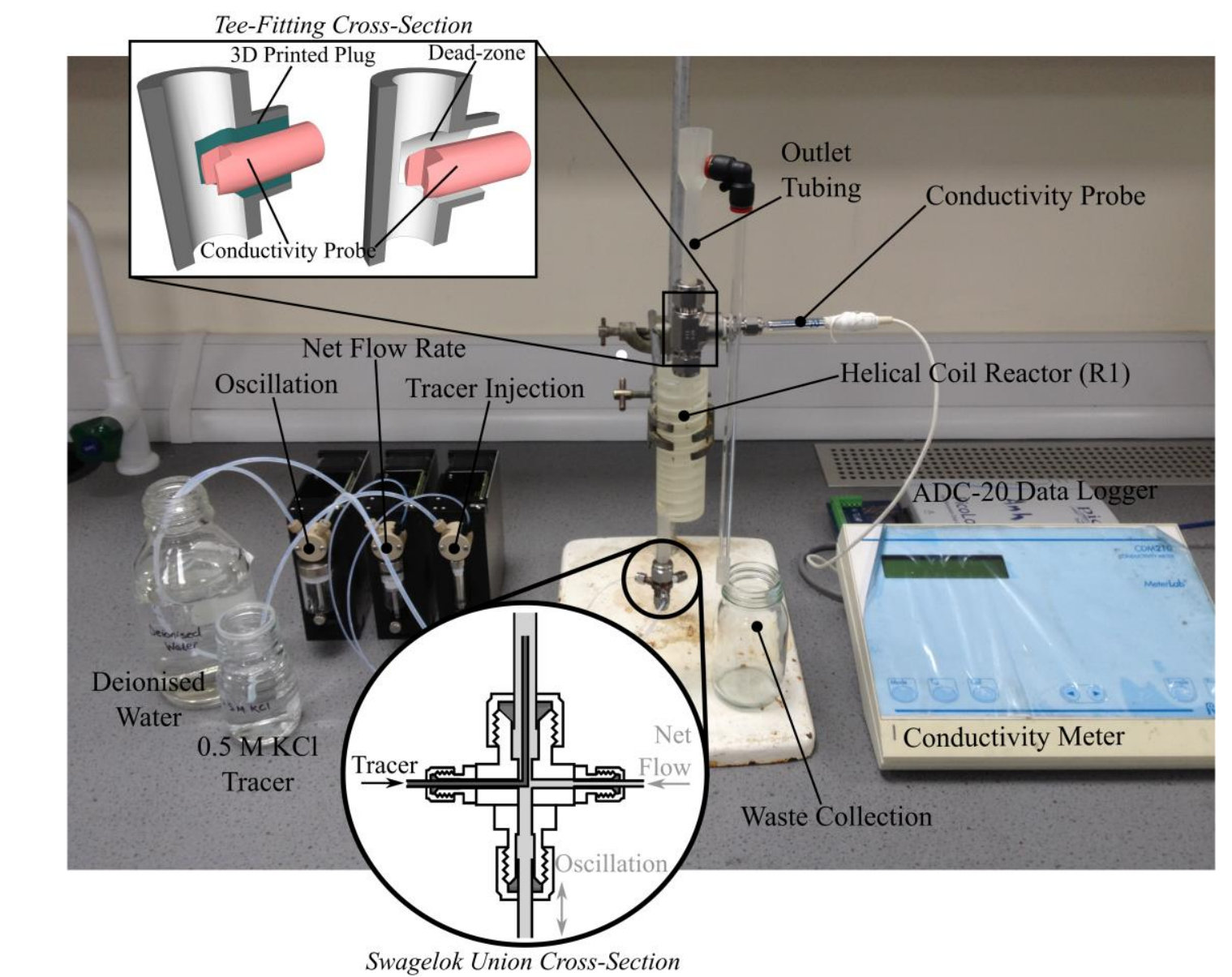

Figure 4. Experiment setup for measuring residence time distributions (RTDs) in helical coil reactor geometries subject to a range of oscillation intensities and net flow rates

The net flow of deionised water, fluid oscillation and salt tracer injection were generated using three syringe pumps (C3000 series, TriContinent), which were all connected to the lower tee-junction via a custom Swagelok union and 1/16 in (net flow and tracer) and 1/8 in (oscillation) PTFE tubing. The oscillatory flow was applied at the base of the union fitting from a reservoir of deionized water using a $12.5 \mathrm{~mL}$ syringe. The oscillation amplitude was controlled using the volume of dispensed liquid, whilst the frequency was controlled by adjusting the speed and acceleration settings of the lead screw. The net flow of deionized water was provided from the same reservoir using a $5 \mathrm{~mL}$ syringe, which provided a good compromise between minimising cavitation during the refill and minimising the number of refills of the syringe required per experiment. Finally, the tracer was injected using a $1 \mathrm{~mL}$ syringe. Here, the tracer was injected just above the net flow inlet point by routing the PTFE tubing through the union (see Figure 4) to avoid dead-zones that would otherwise have produced right-hand skewed RTD curves.

The experiment procedure was as follows. First, the reactor and syringe pumps were purged with deionised water to remove all air from the system. The syringe pumps were then adjusted to the desired net flow rate and oscillation condition and allowed to achieve steady state for 2 minutes. Using the 1 $\mathrm{mL}$ syringe, $0.125 \mathrm{~mL}$ of the $0.5 \mathrm{M} \mathrm{KCl}$ tracer was injected at the base of the reactor in approximately $0.15 \mathrm{~s}$ to provide as close to an ideal pulse as possible. This tracer volume represented just $\sim 0.8 \%$ of the reactor volume, minimising disturbance to the flow, and the time to inject the tracer was approximately three orders of magnitude smaller than the shortest residence time considered in this study $(90 \mathrm{~s})$. Therefore, it can be reasonably assumed that the tracer pulse was narrow enough compared to the mean residence time to approximate a pulse response. The conductivity of the tracer was then measured over time at the outlet, allowing the residence time distribution at each condition to be determined. 


\subsubsection{RTD and Tanks-in-Series Model}

The residence time distribution (RTD) is a collection of the residence times experienced by different fluid elements as they pass through a reactor [37]. In ideal plug flow, all fluid elements spend the same amount of time in the reactor. The resulting RTDs are narrow and symmetric, which is usually the preferred condition for continuous flow reactors. However, fluid elements can take different paths through the reactor as a consequence of non-ideal mixing. This manifests as a broad RTD curve with potential additional non-Gaussian features such as multiple breakthrough peaks (e.g. flow channelling) and high skewness (e.g. dead-zones). By assessing the RTD, insight into the state of the macromixing in the reactor can be ascertained, also allowing for the diagnosis of operational problems.

The exit age distribution, $\mathrm{E}(\mathrm{t})$, quantifies the time spent by different fluid elements in the reactor. For a pulse tracer injection, $\mathrm{E}(\mathrm{t})$ can be defined based on the concentration of tracer $(\mathrm{C})$ using equation 5 . According to this definition, the area under this curve is equal to 1 , meaning the quantity " $\mathrm{E}(\mathrm{t}) \mathrm{dt}$ " describes the fraction of fluid that exits the reactor with a residence time between the particular integration limits [34]. For discrete data, $\mathrm{E}(\mathrm{t})$ is instead defined using equation 6 , where $\mathrm{C}(\mathrm{t})$ is the concentration of tracer measured at time, $\mathrm{t}$, and $\Delta \mathrm{t}$ is the sampling rate. Note, in this study the tracer conductivity measured at the outlet was used directly in equation 6 because there was a linear relationship observed between the conductivity and concentration within the concentration range used. Additionally, the conductivity of the DI water used in this study was $<0.1 \mu \mathrm{S} / \mathrm{cm}$, which was below the detection limit of the conductivity probe meaning there were no offset effects that needed to be removed.

$$
\begin{gathered}
E(t)=\frac{C}{\int_{0}^{\infty} C d t} \\
E(t)=\frac{C(t)}{\sum_{0}^{\infty} C(t) \Delta t}
\end{gathered}
$$

To improve the comparison of each of the reactor geometries, and to facilitate comparison of the results of this study with previous meso-OBR studies, the dimensionless form of the exit age distribution (equation 7) was considered. Here, $\theta$ is the dimensionless time (equation 8 ) and $\tau$ is the mean residence time (equation 9). Thus, for comparable levels of mixing, the normalised $\mathrm{E}(\theta)$ curve will be the same, regardless of the reactor size. Therefore, for good quality plug flow, $\mathrm{E}(\theta)$ will be centred on $\theta=1$, meaning the fluid predominately exits the reactor after approximately one mean residence time.

$$
\begin{gathered}
E(\theta)=\tau E(t) \\
\quad \theta=\frac{t}{\tau} \\
\tau=\frac{\sum t C(t) \Delta t}{\sum C(t) \Delta t}
\end{gathered}
$$

The tanks-in-series model (equation 10) was used to quantify the plug flow performance of each of the reactor geometries. Plug flow is mathematically equivalent to an infinite number of well-mixed stirred tanks connected in series. Consequently, increasing the number of well-mixed fluid volumes in series provides a better approximation to plug flow. The tanks-in-series model is a one-parameter model that describes the plug flow quality using this number of equivalent tanks in series, where $\mathrm{N} \geq 10$ is usually regarded as the minimum acceptable level. For each experiment, $\mathrm{N}$ was determined by matching the model (equation 10) with the $\mathrm{E}(\theta)$ curve measured experimentally using equations $6-9$ so that the shape and height matched. No further statistical moments, such as variance or skewness, were considered.

$$
E(\theta)=\frac{N(N \theta)^{N-1}}{(N-1) !} e^{-N \theta}
$$

\section{Results and Discussion \\ 3.1 Plug Flow Response}

Figure 5 shows four examples of the dimensionless residence time distributions (RTDs) produced by the helical coil reactors in this study when subjected to simultaneous oscillatory and net flow components. At nearly all conditions studied (95\% of the data), at least 10 equivalent tanks-in-series 
was observed, indicating a minimum acceptable level of plug flow was achievable regardless of the operating conditions selected. At certain oscillatory conditions a significant enhancement of the plug flow behaviour was observed, with $\mathrm{N}>100$ produced (see Figure $6 \mathrm{a}$ for example). This enhancement must be due to interaction between the oscillatory fluid motion with the curvature of the tube, because it has been shown that there is no benefit of applying oscillatory motion in straight plain tubes [38]. Furthermore, at low net flow Reynolds numbers $\left(\operatorname{Re}_{\mathrm{n}}=300-400\right)$ with no oscillation, the RTD of coiled tubes is only slightly narrower than uncoiled tubes [7]. Vortex formation and dissipation have been observed in $4.4 \mathrm{~mm}$ diameter mesoscale oscillatory baffled reactors (meso-OBRs) numerically and experimentally at frequencies as high as $11.1 \mathrm{~Hz}$ [39]. This provides precedent that quasi-steady Dean vortices are produced that are not attenuated. Thus, through analogy with the oscillatory baffled reactor, it is likely that Dean vortices are periodically formed then unravelled in the coils when subject to oscillatory motion, validated by Timité et al. [21]. This provides a mechanism to limit axial dispersion through increased radial mixing at lower net flow rates.

(a)

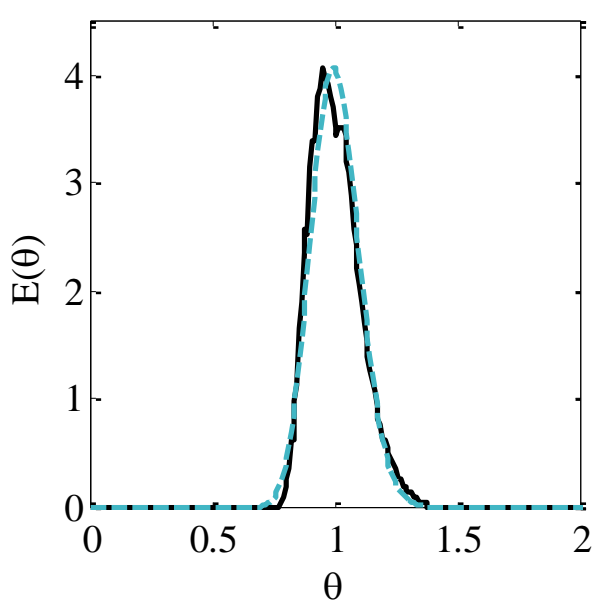

(c)

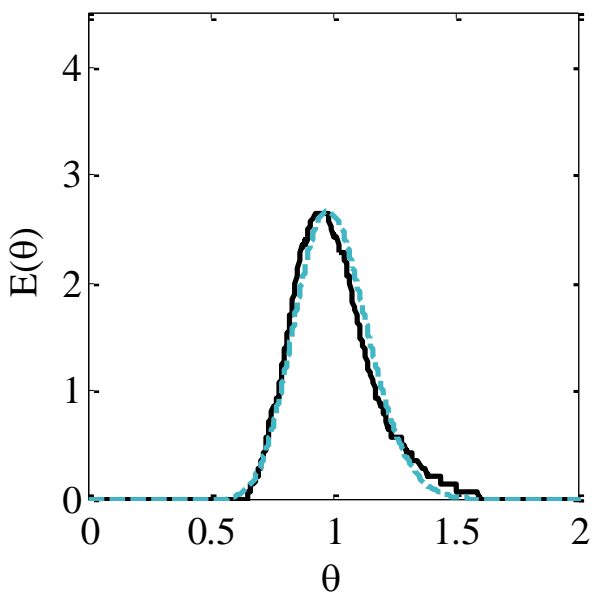

(b)

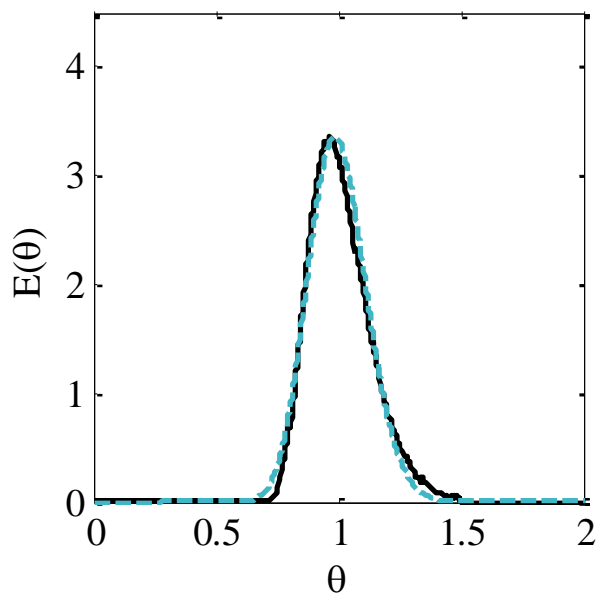

(d)

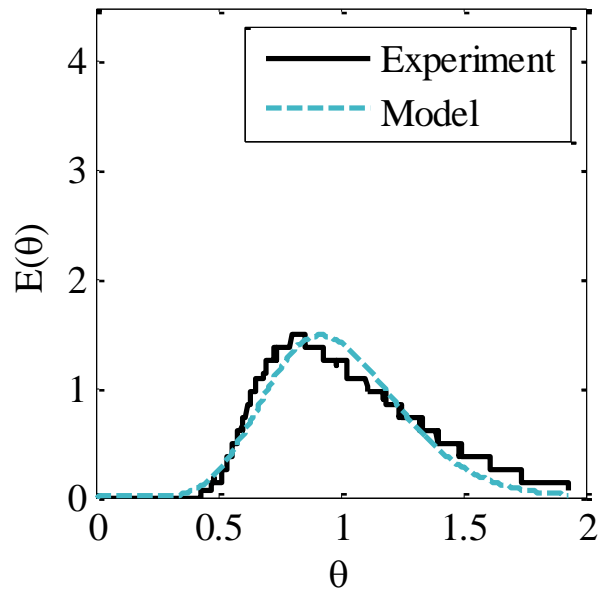

Figure 5. Example dimensionless residence time distribution (RTD) curves showing different plug flow qualities $(N) \mid\left(\right.$ a) $N=\mathbf{1 0 3}$ (reactor geometry $\left.R 1, R_{n}=50, x_{o}=2 \mathrm{~mm}, f=5 \mathrm{~Hz}\right) \mid(b) N=\mathbf{7 0}$ (reactor geometry $\left.R 1, R e_{n}=10, x_{o}=2 \mathrm{~mm}, f=4 \mathrm{~Hz}\right) \mid(c) N=44$ (reactor geometry $R 4, R_{n}=10, x_{o}$ $=2 \mathrm{~mm}, f=5 \mathrm{~Hz}) \mid\left(\right.$ d) $\mathrm{N}=11$ (reactor geometry $R 1, R_{n}=10$, no oscillations)

Although the Dean vortices produced in the helical coil are analogous to the vortices produced in the meso-OBR, the resulting plug flow quality of the oscillatory curved channel flow is superior to the straight-tubed meso-OBR with the same tube diameter $(5 \mathrm{~mm})$. For example, Phan and Harvey [31, $32,40]$ report maximum equivalent tanks-in-series of $\mathrm{N}=25, \mathrm{~N}=20$ and $\mathrm{N}=55$ for central axial, integral and helical baffle designs respectively, at net flows ranging from $\operatorname{Re}_{\mathrm{n}}=2.55$ to 34 . 
Plug flow is mathematically equivalent to an infinite number of infinitesimally small tanks-in-series. Intuitively the space between each baffle in the OBR might be thought of as a well-mixed stirred tank, such that the maximum plug flow quality attainable is generally comparable to the number of these inter-baffle mixing zones in the reactor. However, in the helically coiled channels, the physical interpretation of the number of tanks-in-series is less clear. In the CFI, the mixing zones (physical manifestation of the tanks-in-series) could be related to the Dean vortices that form between the inversions. Similarly, in the helically coiled channels with oscillatory motion, the mixing zones might be linked to the breakdown of the longitudinal vortices during flow reversal, analogous to the breakdown of toroidal vortices in OBRs into elongated 'worm' structures [41].

The vortices produced around the orifice-type constrictions in an OBR are generally toroidal, with their plane of rotation aligning parallel to the main flow. These toroidal vortices are then swept across the inter-baffle zone before unravelling as the flow direction reverses [39]. In contrast, in helically coiled channels the Dean vortices are orientated longitudinally, where the rotation plane of the vortices is orthogonal to the direction of the net flow (see Figures 1 and 2). Therefore, the amount of fluid influenced by the Dean vortices in the coiled channels is likely to be larger than the toroidal vortices produced in the OBR in any one instant. Ultimately, this could improve the global radial mixing rate observed across a full oscillation cycle in the helically coiled channels leading to the improved plug flow response. Interestingly, the helical baffles of the meso-OBR share characteristics with both the helical channel and orifice-like constrictions of standard OBRs, where helically shaped vortices are produced [35]; its plug flow performance is also mid-way between the two.

Ordinarily, the fluid at the centres of the Dean cells can only 'escape' via diffusion [10]. Coiled flow inverters (CFIs) are a well-known approach for improving the mixing/plug flow performance of coiled tubes. The direction of the centrifugal force is always orientated toward the outer wall, meaning when the coil's direction is changed, the plane in which the Dean vortices form also rotates by the same angle. Considering Figure 1b, following a change in direction of the coil, the points previously in the centres of the Dean vortices (where the velocity is a minimum) now coincide with the centreline between the two vortices (where the velocity is a maximum), and vice versa. Thus, the rotation of the plane of the Dean vortices following the change in direction of the bend improves radial mixing [5], by mitigating the effects of fluid trapping regions (diffusion limited mixing) that would otherwise occur at the centres of the Dean vortices without the use of flow inversions [10].

Whilst CFIs disrupt the Dean vortices "in space", the oscillatory fluid motion in this study disrupted the Dean vortices "in time". Here, the continual formation and destruction of the vortices probably prevented the formation of stagnation points, through improved radial mixing [21]. It should be noted that these vortices would form in the same plane during the forward and backward strokes of the oscillation cycle because the direction for centrifugal force remains constant.

It has been shown that the plug flow performance of CFIs improves as the number of bends is increased. For example, Saxena and Nigam [5] found that the F-curves produced from tracer step response experiments approached closer to ideal plug flow as the number of bends was increased from 3 to 57, and that CFIs substantially reduced the axial dispersion rate compared to helically coiled tubes. Kurt $e t$ al. [17] recently confirmed this result, showing that increasing the number of inversions was the critical design parameter for minimising axial dispersion. However, Saxena and Nigam [5] also noted that for fixed reactor volumes/diameters, there is a restriction on the maximum number of turns that can be applied, because a minimum number of turns of the coil between each inversion is needed for the development of the secondary flow structures. Therefore, one advantage for the use of oscillatory motion is that the equivalent of 57 "time-inversions" could be implemented in just $11.4 \mathrm{~s}$ when using a $5 \mathrm{~Hz}$ oscillation frequency. This means there is potentially a higher limit to the number of equivalent flow inversions that can be produced practically (for a give mean residence time). Similar to the OBR, oscillatory motion in the helical coil decouples the mixing behaviour from the net flow rate, allowing longer mean residence times to be achieved using smaller reactor lengths. 


\subsection{Effect of Operating Conditions}

\subsubsection{Oscillation Amplitude and Frequency}

Figure 6 shows the effects of frequency and amplitude on the plug flow response at three different net flow rates $\left(\operatorname{Re}_{\mathrm{n}}=10,30\right.$ and 50$)$. When increasing the oscillation intensity, there appeared to be a 'switch-on' followed by 'switch-off' of the plug flow enhancement. For example, using oscillation amplitudes of $1 \mathrm{~mm}$ and $8 \mathrm{~mm}$ (Figures 6a, 6c and 6e), the number of equivalent tanks-in-series ranged from 7-20. One explanation for the reduced plug flow performance observed for the $8 \mathrm{~mm}$ amplitude is the axial dispersion rate increases faster than the radial mixing rate because the Dean vortex sizes are limited by the diameter of the tube. The $8 \mathrm{~mm}$ amplitude produces oscillatory Dean numbers of $\mathrm{De}_{\mathrm{o}} \geq$ 224 (when $x_{0} \geq 2 \mathrm{~mm}$ ), which exceeds the conditions at which stable secondary Dean vortex instabilities are expected to occur for steady flows (De > 200), resulting in more chaotic mixing. The lack of any significant enhancement for the $1 \mathrm{~mm}$ amplitude can also be explained using the Dean number. As discussed in the next section, the $1 \mathrm{~mm}$ amplitude produced oscillatory Dean numbers of $\mathrm{De}_{\mathrm{o}}<70$, which is below the threshold for the onset of the Dean instabilities in steady flow [4]. As a result, the optimum frequency (highest number of equivalent tanks-in-series), shifted to smaller values as the amplitude increased. Larger oscillation frequencies are required to compensate for the smaller amplitudes to keep the oscillatory Dean number in the range of $\mathrm{De}_{0}=70-200$ [4].

Oscillation amplitudes of 2-4 mm caused significant increases in the plug flow performance compared to the $1 \mathrm{~mm}$ and $8 \mathrm{~mm}$ amplitudes, achieving maximum equivalent tanks-in-series of 50-100. Here, there was also an optimal frequency for plug flow at each amplitude. The optimal frequency range was typically around 2-6 Hz, with the optimal frequency increasing as $\mathrm{Re}_{\mathrm{n}}$ increased. For example, using $\mathrm{x}_{\mathrm{o}}=2 \mathrm{~mm}$ the optimal frequencies were $3 \mathrm{~Hz}$ at $\operatorname{Re}_{\mathrm{n}}=10$ (Figure 6a) and $6 \mathrm{~Hz}$ at $\operatorname{Re}_{\mathrm{n}}=50$ (Figure 6e). The optimum frequency range coincided with oscillatory Dean numbers of 70-200 (further discussed in the next section), implying that these frequencies were suitable for the formation of stable Dean vortices. Thus, $\mathrm{De}_{\mathrm{o}}=70-200$ roughly defines the operating window for plug flow in these coiled tubes subject to oscillatory motion. 
(a)

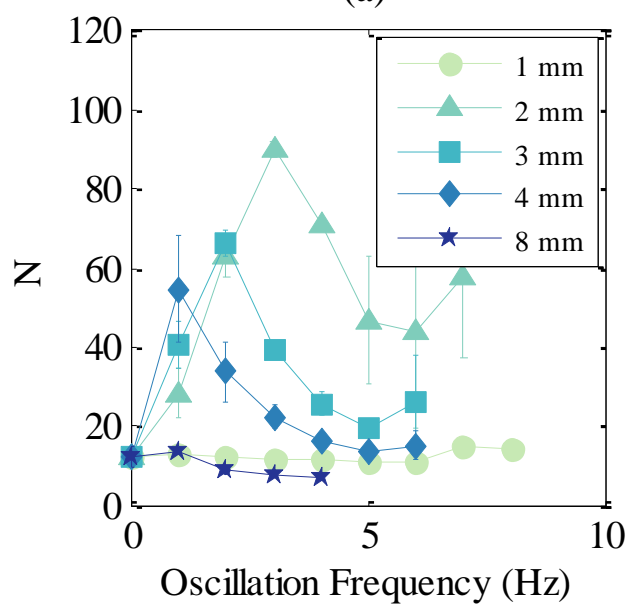

(c)

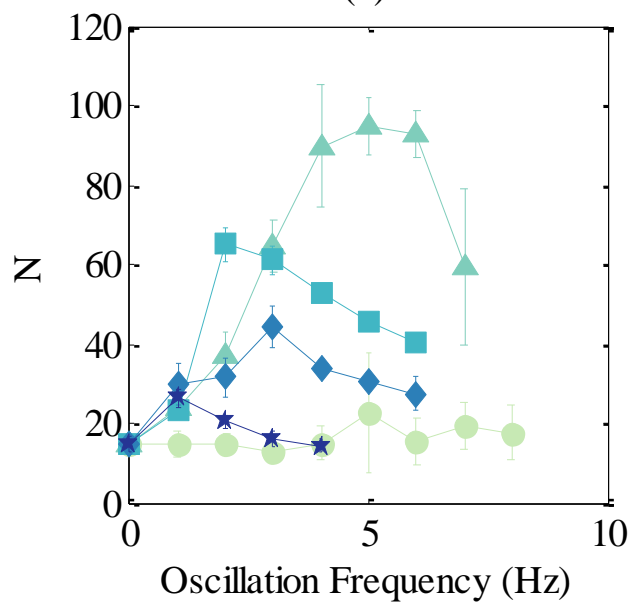

(e)

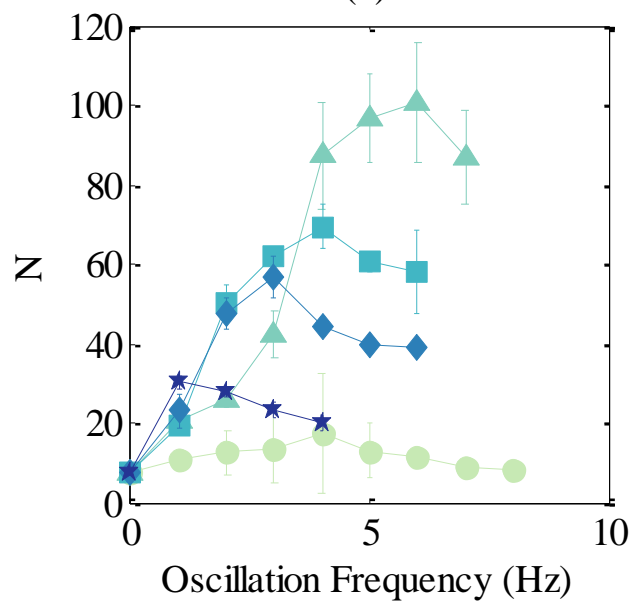

(b)

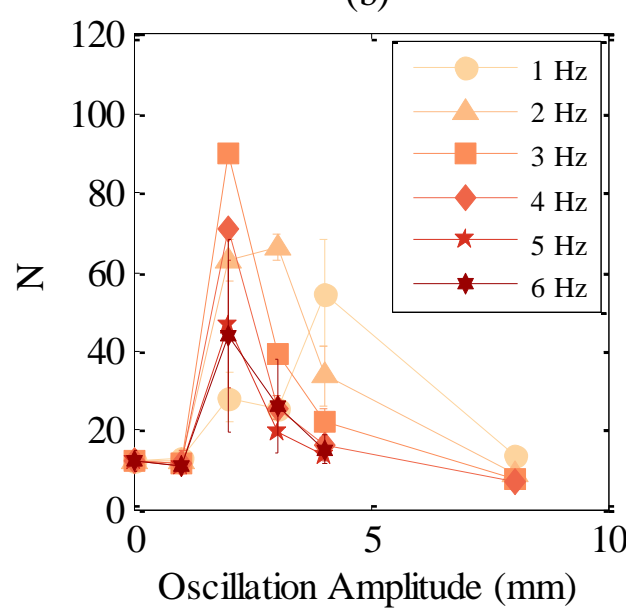

(d)

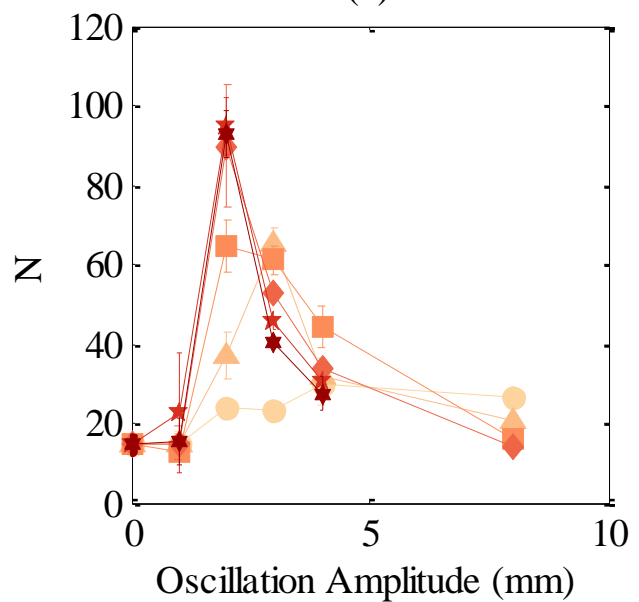

(f)

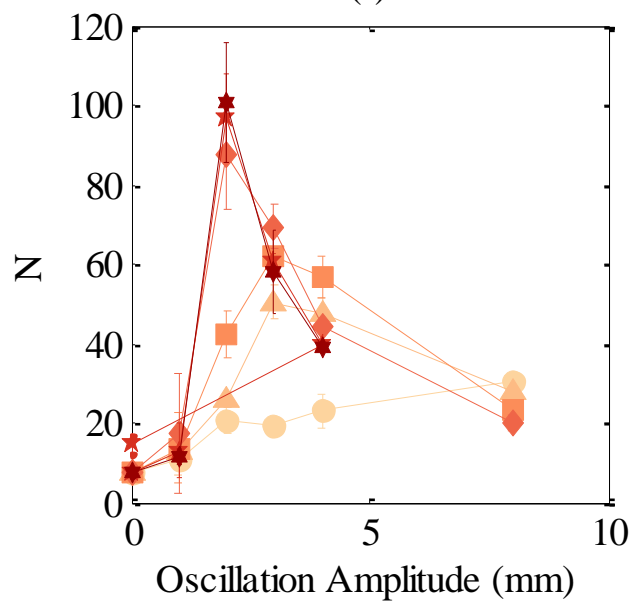

Figure 6. Effect of oscillation frequency $(f)$ and oscillation amplitude $\left(x_{o}\right)$ on the plug flow behaviour $(N)$ at different net flow Reynolds numbers $\left(R e_{n}\right)\left|(a-b) R e_{n}=10,(c-d) R e_{n}=30,(e-f) R e_{n}=50\right|$ reactor geometry $R 1$ (see Table 1)

In Figure 6, it can be clearly seen that for reactor geometry R1, the $2 \mathrm{~mm}$ amplitude was favourable in the optimal frequency range, producing a significant enhancement of the plug flow quality compared to all other amplitudes (up to $\mathrm{N}=100$ ). Further discussion is provided in Section 3.3.2, where the optimal amplitude observed changed as a function of the radius of curvature. 


\subsubsection{Flow Rate and Oscillatory Dean Number}

As mentioned in the methodology, an "oscillatory Dean number" (equation 4) has been proposed for this study to characterise the effect of oscillation intensity on the fluid mechanics whilst taking into account the curvature of the channels. Figure 7 shows the effect of $\mathrm{De}_{\mathrm{o}}$ on the plug flow response for reactor geometry $\mathrm{R} 1$ at an amplitude of $2 \mathrm{~mm}$ at five different flow rates.

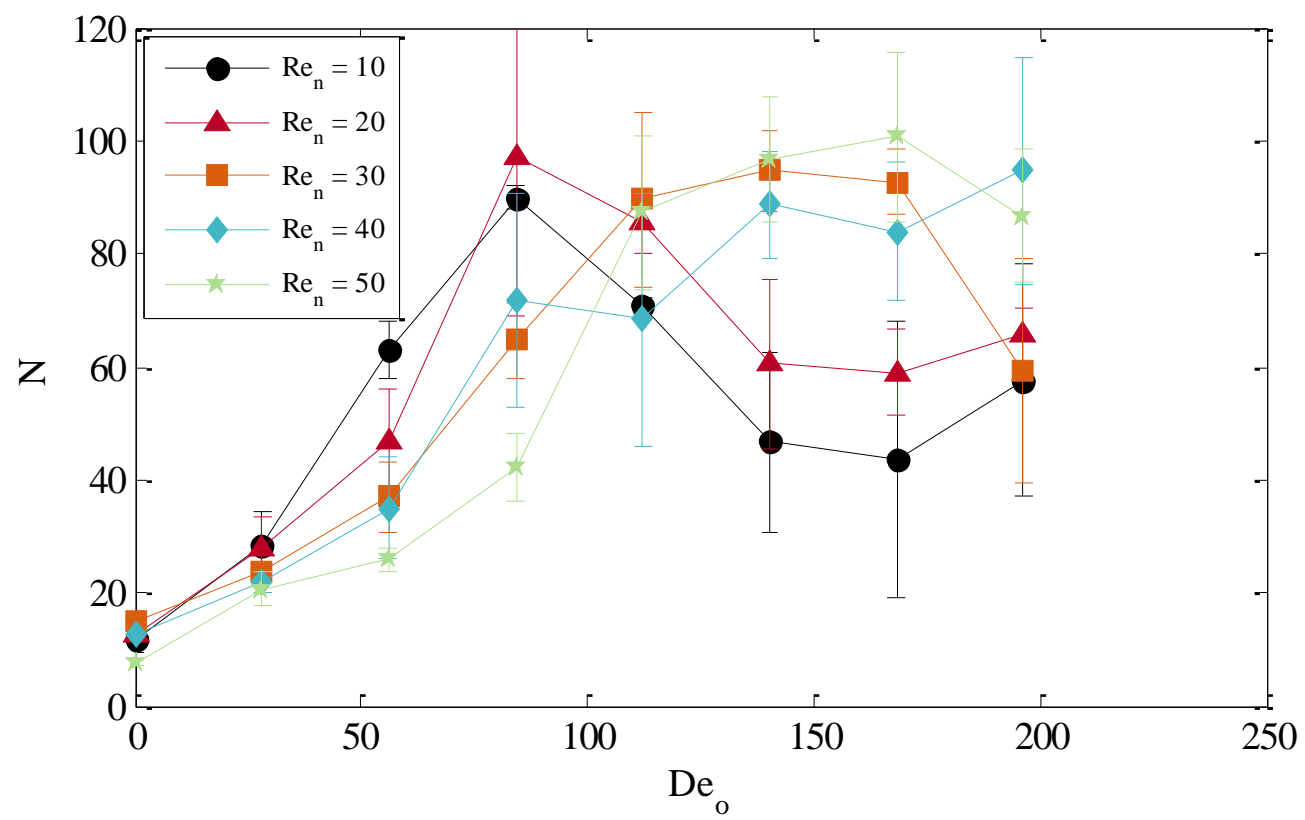

Figure 7. Effect of oscillatory Reynolds number $\left(R e_{o}\right)$ on the plug flow behaviour $(N)$ at different net flow Reynolds numbers $\left(\operatorname{Re}_{n}\right)$ at optimum oscillation amplitude $\left(x_{o}=2 \mathrm{~mm}\right) \mid$ reactor geometry $R 1$

In Figure 7, the oscillation amplitude was maintained at a constant value of $2 \mathrm{~mm}$, meaning the oscillatory Dean number was adjusted using just the oscillation frequency. At low $\operatorname{Re}_{\mathrm{n}}$ and low oscillation frequencies, the total velocity during the forward and backward phases of the oscillation cycle (upstroke and downstroke of the oscillator) was small, meaning there was insufficient flow to generate the Dean instability. Then as the frequency increased, the total velocity also increased leading to the formation of Dean vortices. The maximum observed in Figure 7 can be attributed to a transition point between radial and axial mixing promotion; the radial mixing rate is limited by the growth of the Dean vortices (vortices become bounded by the channel wall $[23,35]$ ) whereas the growth of axial mixing is not constrained. Thus, if the oscillation intensity is too high, the radial mixing generated by the vortices will be insufficient to counteract the increased axial dispersion.

In the range studied, $\operatorname{Re}_{\mathrm{n}}$ had minimal effect on the number of equivalent tanks-in-series produced by each reactor design. Instead, increasing $\mathrm{Re}_{n}$ only increased the value of $\mathrm{De}_{\mathrm{o}}$ at which the maximum number of tank-in-series occurred (see Figure 7). Here, the peak plug flow quality was still observed in the range of $\mathrm{De}_{\mathrm{o}}=70-200$ for all designs, which corresponds to the region where secondary Dean instabilities are expected to form [4]. Net flow Reynolds numbers of $\operatorname{Re}_{\mathrm{n}}=10$ and $\mathrm{Re}_{\mathrm{n}}=50$ correspond to net velocities of $\sim 2 \mathrm{~mm} / \mathrm{s}$ and $\sim 10 \mathrm{~mm} / \mathrm{s}$. Therefore, at the higher net flow Reynolds numbers, the magnitude of the oscillatory velocity is diminished during the backward phase of the oscillation cycle. This is illustrated in Figure 8. Although stronger vortices will be produced during the forward phase of the oscillation cycle, these do not compensate for the loss of vortex formation during the backward phase of the cycle; the net effect over the full cycle is reduced radial mixing and increased axial dispersion. Consequently, higher flow rates require a higher frequency to ensure consistent vortex formation across the full oscillation cycle, resulting in a shift in the value of $\mathrm{De}_{\mathrm{o}}$ in which the maximum $\mathrm{N}$ occurs. 
(a)

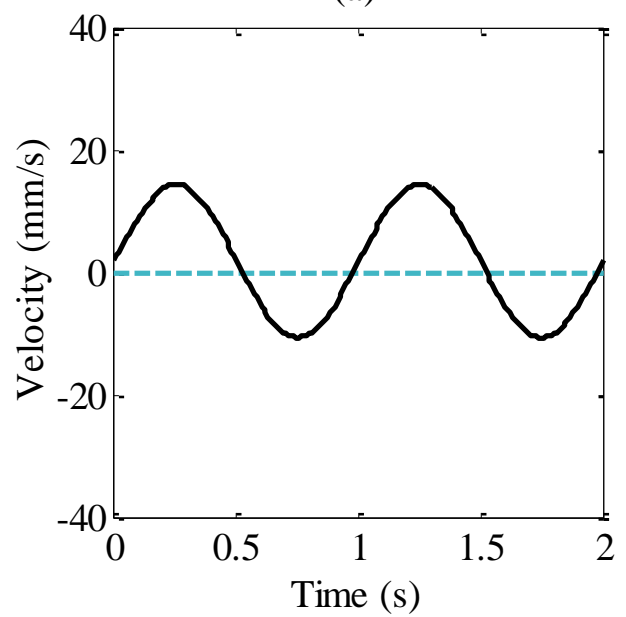

(c)

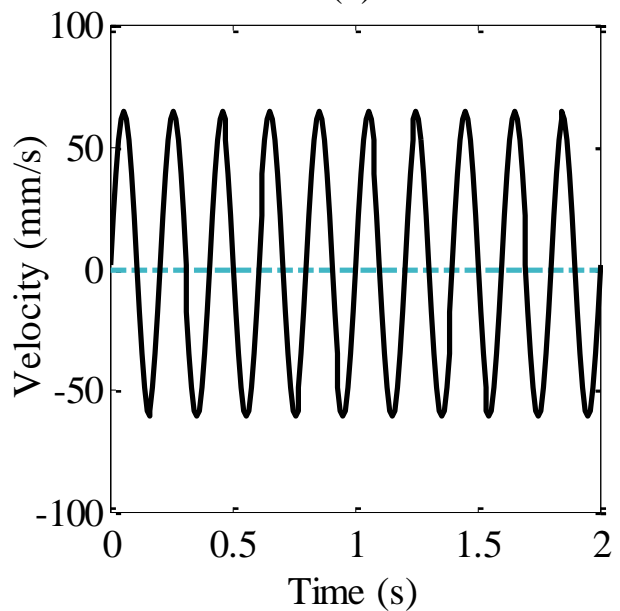

(b)

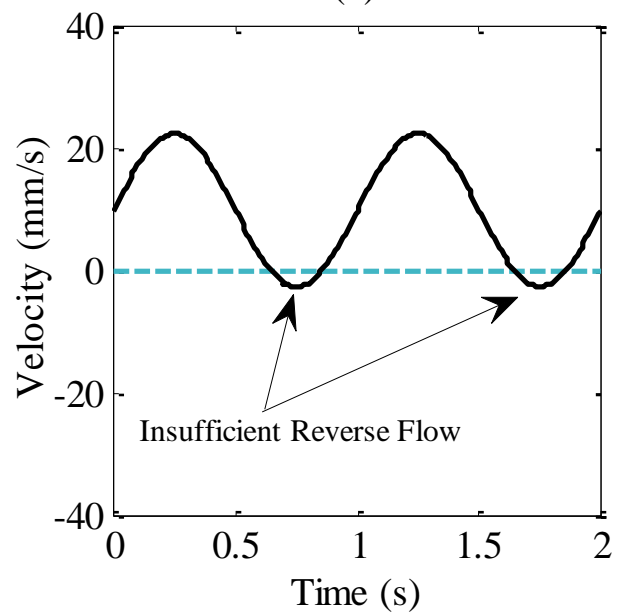

(d)

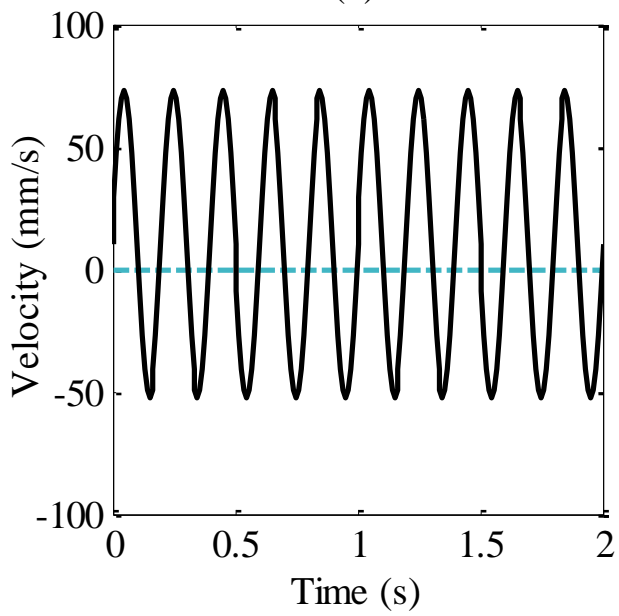

Figure 8. Total inlet velocity (oscillation + net flow) at different net flow Reynolds numbers and oscillation frequencies $\left|x_{o}=2 \mathrm{~mm}\right|(\mathrm{a}) \operatorname{Re}_{n}=10(\mathrm{v}=2 \mathrm{~mm} / \mathrm{s}), f=1 \mathrm{~Hz} \mid(\mathrm{b}) \operatorname{Re}_{n}=50(v=10 \mathrm{~mm} / \mathrm{s})$, $f=1 \mathrm{~Hz} \mid$ (c) $R e_{n}=10(v=2 \mathrm{~mm} / \mathrm{s}), f=5 \mathrm{~Hz} \mid$ (d) $R e_{n}=50(v=10 \mathrm{~mm} / \mathrm{s}), f=5 \mathrm{~Hz}$

Nevertheless, good plug flow $(\mathrm{N} \geq 50)$ could be produced at net flow Reynolds numbers as low as $\mathrm{Re}_{\mathrm{n}}$ $=10$ provided the correct oscillation conditions are selected. This is a significant reduction of $\operatorname{Re}_{\mathrm{n}}$ to produce plug flow compared with helical coil reactors, where initial mixing enhancements were only observed for $\operatorname{Re}_{\mathrm{n}}>300$ [7]. More recently, Sharma et al. [9] report that helical coils containing zero to three inversions have a design advantage compared with straight tubes for $\operatorname{Re}_{\mathrm{n}}>70$ (though $\operatorname{Re}_{\mathrm{n}}>200$ would be required for one order of magnitude improvement), whilst Klutz et al. [42] report plug flow behaviour in CFIs as low as $\operatorname{Re}_{n}=24$ following a detailed parametric study. More generally, Rossi et al. [15] observed that CFIs have a design advantage compared to helically coiled channels with no inversions for $\mathrm{De}>2$, equivalent to $\mathrm{Re}_{\mathrm{n}}>15$. Thus, the use of oscillatory flow in the helical coil geometries is at least comparable with the established CFI. Compared to the steady flow results of Koutsky \& Adler [7] and Sharma et al. [9], oscillatory motion would allow reactors to be 30 and 7-20 times shorter respectively. Although not explored in the present study, there is an evident opportunity to test whether the combination of CFI with oscillatory motion would result in further enhancement of the plug flow. The lower $\mathrm{Re}_{\mathrm{n}}$ limit for plug flow using oscillatory motion should also be established: enabling plug flow at lower flow rates would minimise waste generation and extend the applicability of the reactor platform to longer mean residence time processes.

The variance of the RTD, $\sigma^{2}$, is related to the equivalent number of tanks-in-series, $\mathrm{N}$, according to equation 11 [43]. In this study, the reactor was behaving as an open vessel, where dispersion of the tracer could take place upstream (between the tracer injection point and reactor inlet) and downstream 
(after the tracer detection point). For this open-open boundary condition, it can be shown that the Péclet number is related to the variance, $\sigma^{2}$, and mean residence time, $\tau$, using equation 12 [43]. The Péclet number, Pe, (equation 13) describes the ratio of the convective transport rate to diffusive transport rate; larger values of Pe indicate better plug flow performance. In equation 13, $\mathrm{U}$ is the characteristic velocity, $\mathrm{L}$ is the characteristic length and $\mathrm{D}_{\mathrm{f}}$ is the diffusivity. Thus, it can be seen that the Péclet number is related to the number of tanks-in-series using equation 14.

$$
\begin{gathered}
N=\frac{\tau^{2}}{\sigma^{2}} \\
\frac{\sigma^{2}}{\tau^{2}}=\frac{2}{P e}+\frac{8}{P e^{2}} \\
P e=\frac{U L}{D_{f}} \\
N=\frac{P e^{2}}{2 P e+8}
\end{gathered}
$$

The maximum average number of equivalent tanks-in-series produced in this study was 101, obtained in reactor design $\mathrm{R} 1$ using $\operatorname{Re}_{\mathrm{n}}=50(\mathrm{v}=10 \mathrm{~mm} / \mathrm{s}), \mathrm{f}=6 \mathrm{~Hz}$ and $\mathrm{x}_{\mathrm{o}}=2 \mathrm{~mm}$. Using equation 14, this gives $P e=205.9$ (discounting the negative root). Design 1 had a curvature ratio $\left(\lambda=2 R_{c} / D\right)$ of $\lambda=5$. For a similar curvature ratio of $\lambda=7.6$, Sharma et al. [9] obtained the same Pe using steady flows in a helical coil using $\operatorname{Re}_{\mathrm{n}} \approx 8300$, and a CFI containing three inversions using $\operatorname{Re}_{\mathrm{n}} \approx 6400$. For the lowest net flow rates considered by Sharma et al. [9] $\left(\mathrm{Re}_{\mathrm{n}}=68.8\right)$ Péclet numbers of just 10 were obtained. This shows that oscillatory motion substantially improves the plug flow performance of these reactors in the laminar regime compared to both steady flow helically coiled channels and steady flow CFIs.

\subsubsection{Velocity Ratio}

The velocity ratio (defined as $\psi=\mathrm{Re}_{\mathrm{o}} / \mathrm{Re}_{\mathrm{n}}$ ) has been widely used to describe the interaction between net flow and oscillatory motion in OBRs [31,32,44]. Thus, in the present study, a modified velocity ratio (equation 15) was defined in order to characterise the effect of $\operatorname{Re}_{n}$ observed in Figure 7. Figure 9a shows this modified velocity ratio plotted against the number of equivalent tanks-in-series. It can be seen that the optimal ratio was around 2-8 for amplitudes of $x_{0} \geq 2 \mathrm{~mm}$, and the effect of amplitude observed in Figure 6 is also apparent.

$$
\psi^{\prime}=\frac{D e_{o}}{R e_{n}}
$$

(a)

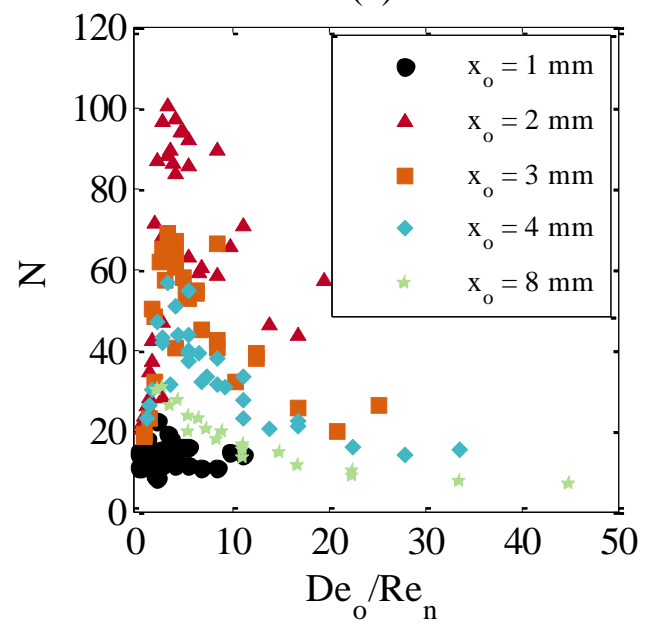

(b)

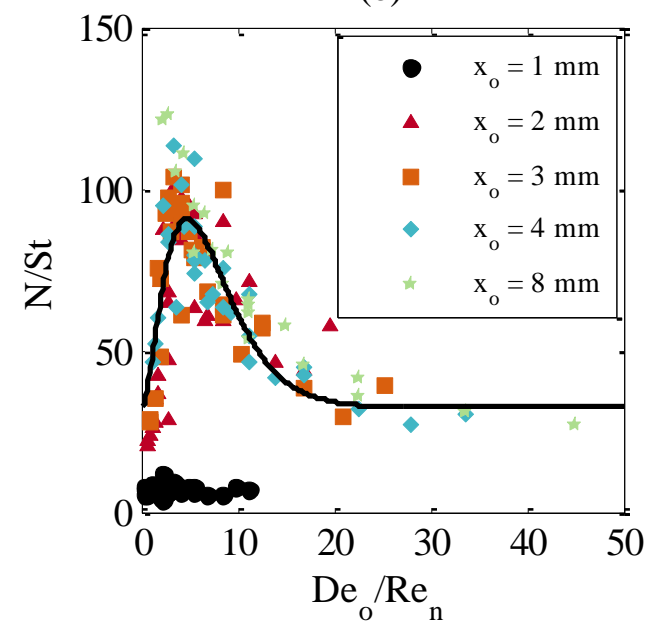

Figure 9. (a) Relationship between plug flow behaviour ( $N$ ) and modified velocity ratio ( $\psi^{\prime}=$ $\left.D e_{o} / R e_{n}\right)$ at different oscillation amplitudes $\left(x_{o}\right) \mid(b)$ relationship between N/St and modified velocity ratio $\left(\psi^{\prime}=D e_{d}\left(R_{n}\right)\right.$ at different oscillation amplitudes $\left(x_{o}\right) \mid$ reactor geometry $R 1$ 
One way to reconcile the different plug flow qualities produced by each amplitude is to take into consideration the Strouhal number. The Strouhal number characterises oscillatory flows, and is often used to describe vortex shedding phenomena. This dimensionless group contains a characteristic frequency (f), characteristic length/spatial dimension (L) and characteristic velocity (U). By replacing these with the oscillation frequency (f), radius of curvature $\left(R_{c}\right)$ (justified in Section 3.3.2) and maximum oscillatory velocity $\left(2 \pi \mathrm{fx}_{\mathrm{o}}\right)$ respectively, equation 16 is obtained. As shown in Figure $9 \mathrm{~b}$, when the modified velocity ratio $\left(\mathrm{De}_{\mathrm{o}} / \mathrm{Re}_{\mathrm{n}}\right)$ is plotted against $\mathrm{N} / \mathrm{St}$, the data for $\mathrm{x}_{\mathrm{o}} \geq 2 \mathrm{~mm}$ roughly collapses onto a single design curve (equation $17, \mathrm{R}^{2}=62.2 \%$ ). Here, the optimal range of $\mathrm{De}_{\mathrm{o}} / \mathrm{Re}_{\mathrm{n}}$ (where N/St is maximised) is apparent.

$$
\begin{gathered}
S t=\frac{f L}{U}=\frac{R_{c}}{2 \pi x_{o}} \\
N / S t=32.44+19.18 \psi^{\prime 1.98} e^{-0.41 \psi^{\prime}}
\end{gathered}
$$

It should be noted that equation 17 is only applicable to the range of conditions studied (Geometry R1, $\mathrm{De}_{\mathrm{o}}=9-449, \mathrm{Re}_{\mathrm{n}}=10-50$ ). Also, the low $\mathrm{R}^{2}$ is likely to be a consequence of the non-linear sensitivity of the tanks-in-series model. I.e. $\mathrm{N}$ is sensitive to small errors in the data/model fit when $\mathrm{N}$ is large; a difference of 10 tanks when $\mathrm{N}=90-100$ might be difficult to discern in the data. Whereas, the difference between $\mathrm{N}=5$ and $\mathrm{N}=15$ would be more significant. Nevertheless, this correlation adequately describes the location of the optimal modified velocity ratio observed in the data. Additional/more complex terms were not considered in this model to avoid over-fitting, where noise captured by the model would reduce its robustness.

In the OBR, the Strouhal number is used to characterise vortex propagation behaviours. Smaller values of St indicate that the forcing velocity (maximum oscillatory velocity) is more dominant, resulting in vortices being pushed further downstream during the oscillation cycle. Larger Strouhal numbers conversely correlate to situations where viscosity dominates the flow (the amplitude is insufficient to overcome the dissipation provided by the viscosity), meaning there is less intense vortex formation. In Section 3.2.1, it was suggested that the $8 \mathrm{~mm}$ amplitude might promote axial dispersion more than radial mixing because the Dean vortices only have limited room to grow owing to the constraint provided by the tube wall (whereas there is no constraint in the axial direction). A similar phenomenon is reported for the meso-OBR [35] and OBR [23]. Consequently, according to equation 16, smaller Strouhal numbers describe more dominant axial mixing compared to radial mixing in the helical coil geometries. The ratio of N/St can therefore be thought of as a normalisation of the plug flow behaviour according to the relative intensity of radial and axial mixing.

In Figure 9b, it can be seen that the $1 \mathrm{~mm}$ amplitude behaved fundamentally differently to the other oscillation amplitudes, where no significant improvement of the plug flow was observed using any frequency or net flow rate compared to the steady flow results. The oscillatory Dean number range at this amplitude was $D_{0}=14-112$, which as previously discussed, suggest that these conditions may have been insufficient to generate stable Dean vortices [4].

\subsection{Effect of Tube Geometry}

\subsubsection{Pitch}

The pitch of a helical tube defines the compactness of the coil. A straight tube can be thought of as having an infinite pitch, whilst zero pitch defines a circular path (i.e. toroidal); thus, larger pitches describe more loosely wound coils, with the 'tightness limit' equal to the outer diameter of the tube. Figure 10 compares the effects of three different tube pitches on the plug flow performance in the presence of oscillatory motion at two net flow Reynolds numbers and two oscillation amplitudes using a radius of curvature of $12.5 \mathrm{~mm}$. At $\operatorname{Re}_{\mathrm{n}}=10$ (Figures $10 \mathrm{a} \& 10 \mathrm{c}$ ), there is no discernible effect of tube pitch on the plug flow behaviour at amplitudes of $2 \mathrm{~mm}$ and $4 \mathrm{~mm}$, agreeing with the observation that the pitch does not have a significant effect on the tube friction factor if the change in pitch is less than the radius of curvature [44]. Similarly, for $\operatorname{Re}_{\mathrm{n}}=50$ and $\mathrm{x}_{\mathrm{o}}=4 \mathrm{~mm}$ (Figure 10d), there was little difference in the plug flow quality between each design. 
However, for $\operatorname{Re}_{\mathrm{n}}=50$ and amplitude of $2 \mathrm{~mm}$ (Figure 10b), a more significant deviation in the plug flow behaviour was observed that was not accounted for by random experiment error. Here, the results for the $7.5 \mathrm{~mm}$ and $12.5 \mathrm{~mm}$ pitched designs matched, whilst the $10 \mathrm{~mm}$ pitch exhibited better plug flow quality, with the peak of $\mathrm{N}$ occurring at a slightly higher $\mathrm{De}_{\mathrm{o}}$. Considering that the $10 \mathrm{~mm}$ pitch design was an intermediary between the other two designs, the difference in performance at this flow rate cannot be explained when considering the fluid mechanics. Instead, this apparent anomaly might be explained via defects in the $3 \mathrm{D}$ printed reactors, not related to the surface roughness, but this could not be confirmed in the present study.

Nonetheless, pitch is not a component of the Dean number, and is therefore not expected to influence the Dean instability, which is believed to account for the plug flow behaviour observed using these oscillating flows. Tang et al. [45] numerically studied the flow patterns in various helical coil geometries. For circular cross-sections, they found that increasing the pitch only caused the plane in which the Dean vortices formed to rotate slightly, which is likely to be related to the inversion phenomenon observed in CFIs; qualitatively, the size of the Dean vortices was not significantly affected. Based on this, and the findings from this study and other studies [18, 46], it can be concluded that the pitch has no significant effect on the plug flow performance when oscillatory flow is also applied to the fluid, at least for the case where $\mathrm{P} \leq \mathrm{R}_{\mathrm{c}}$.

(a)

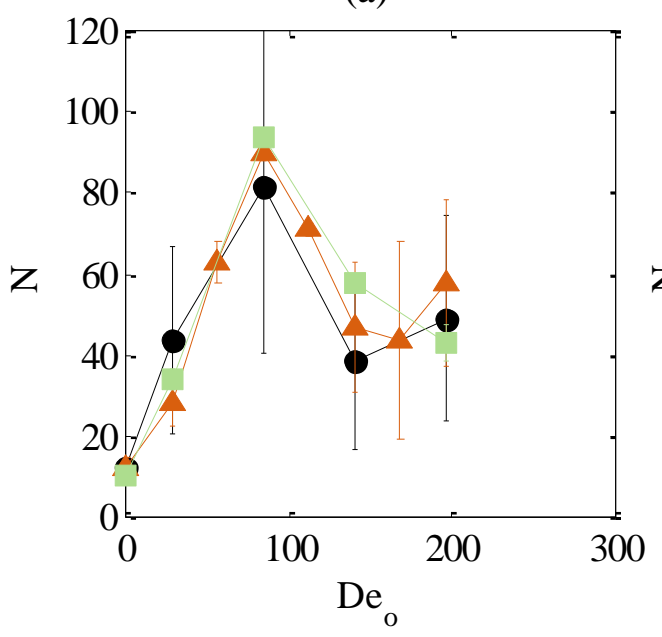

(c)

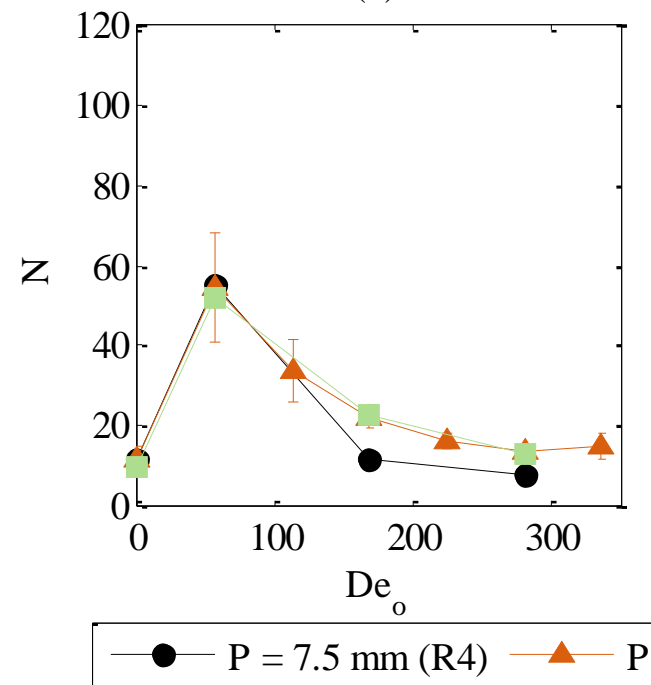

(b)

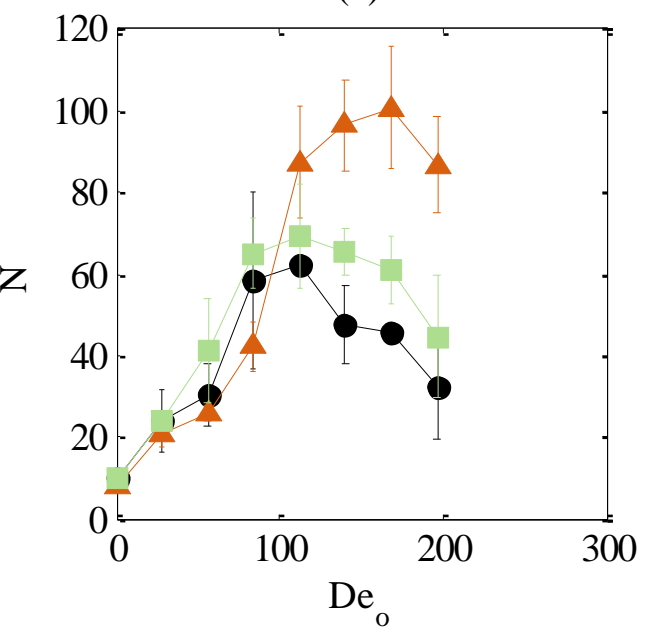

(d)

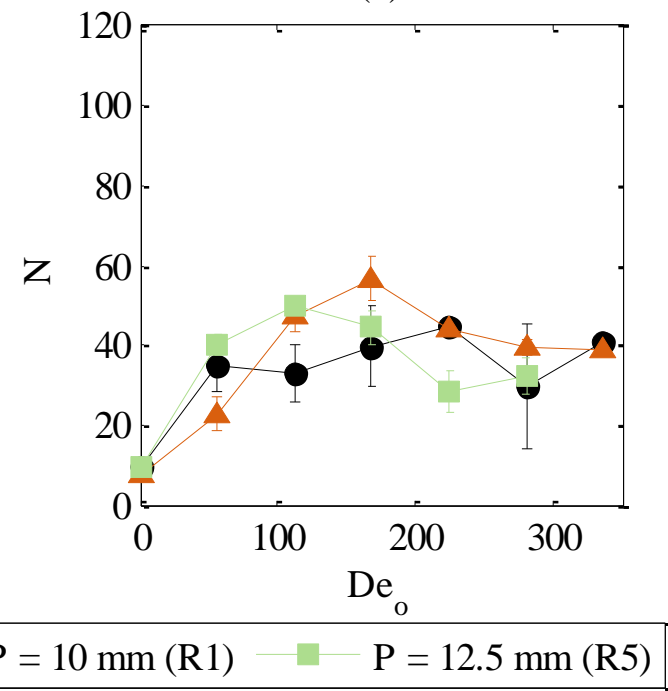

Figure 10. Effect of tube pitch $(P)$ on the plug flow behaviour $(N)$ at different net flow Reynolds numbers $\left(R_{n}\right)$ and oscillation amplitudes $\left(x_{o}\right) \mid(a) R e_{n}=10 \& x_{o}=2 \mathrm{~mm}$, (b) $R_{n}=50 \& x_{o}=2 \mathrm{~mm}$, (c) $R e_{n}=10 \& x_{o}=4 \mathrm{~mm}$ and (d) $R e_{n}=50 \& x_{o}=4 \mathrm{~mm}$ 


\subsubsection{Radius of Curvature}

In contrast to the tube pitch, the radius of curvature of the tube is a component of the Dean number. Smaller radii of curvature to tube diameter ratios $\left(\mathrm{R}_{\mathrm{c}} / \mathrm{D}_{\mathrm{t}}\right)$ produce larger Dean numbers, and hence are expected to produce stronger secondary flows (via stronger centrifugal forces). This has been demonstrated in previous studies, where smaller ratios of $R_{c} / D_{t}$ improve the mixing efficiency (defined as the average deviation of the fluid from complete mixing) [47]. Tang et al. [45] also showed that the vortex centres move closer to the walls as the radius of curvature is decreased.

Figure 11 shows the effects of three radii of curvature $(12.5 \mathrm{~mm}, 22.5 \mathrm{~mm}$ and $32.5 \mathrm{~mm})$ on the plug flow quality. For reactor geometry $\mathrm{R} 1\left(\mathrm{R}_{\mathrm{c}}=12.5 \mathrm{~mm}\right)$, the optimum oscillation amplitude was $2 \mathrm{~mm}$, shown clearly in Figure 6. However, as the radius of curvature increased, the optimal amplitude for plug flow was also found to increase. Here, the optimal amplitude increased from $2 \mathrm{~mm}$ to $4 \mathrm{~mm}$ as the radius of curvature was increased from $12.5 \mathrm{~mm}$ through $22.5 \mathrm{~mm}$ to $32.5 \mathrm{~mm}$. This observation was the reasoning behind the definition of the Strouhal number shown in equation 10.

(a)

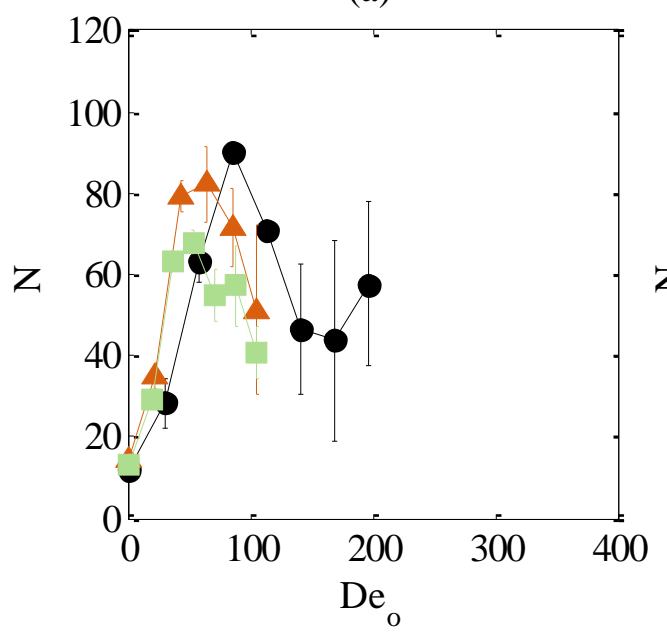

(c)

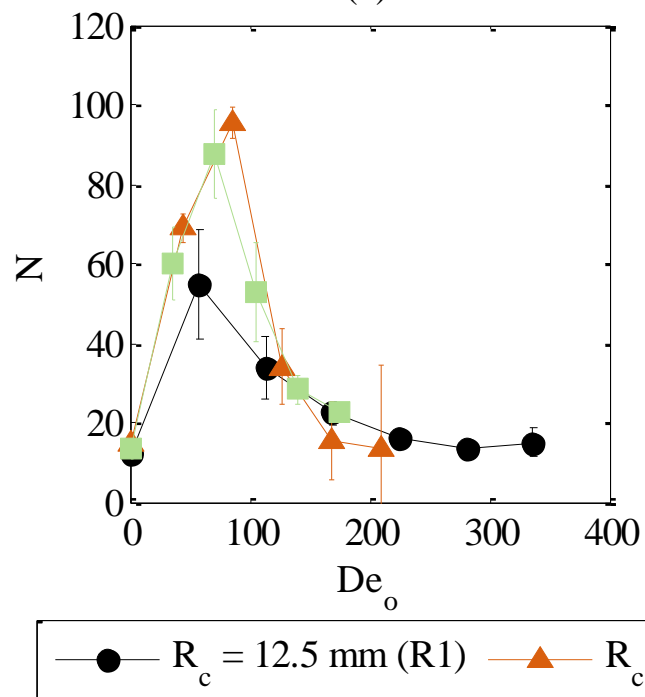

(b)

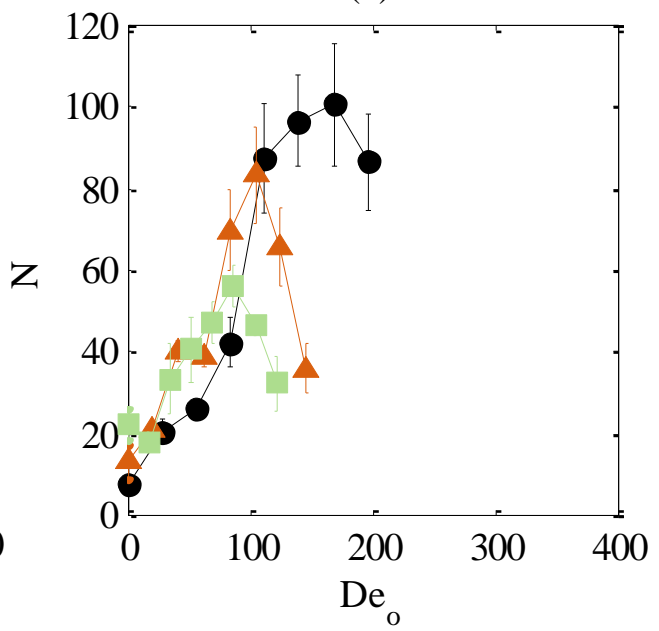

(d)

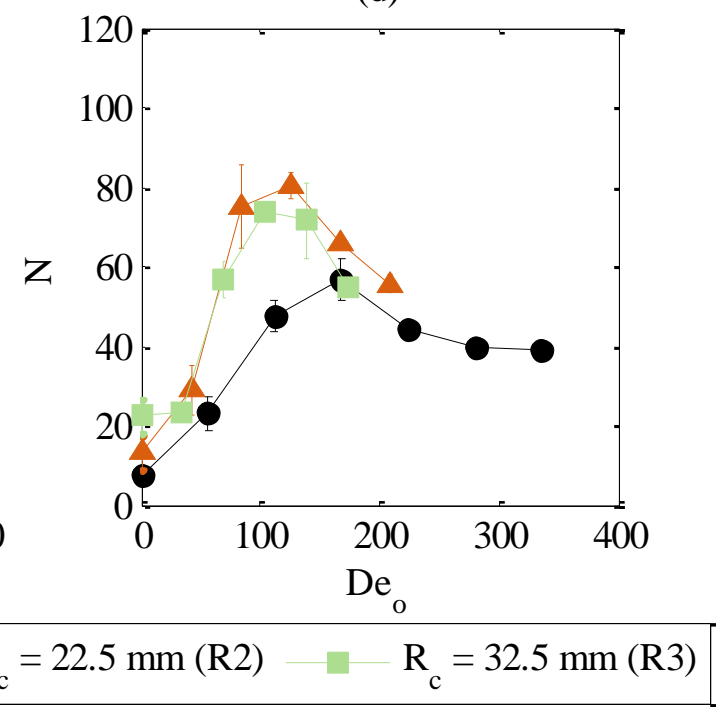

Figure 11. Effect of radius of curvature $\left(R_{c}\right)$ on the plug flow behaviour $(N)$ at different net flow Reynolds numbers $\left(\operatorname{Re}_{n}\right)$ and oscillation amplitudes $\left(x_{o}\right) \mid(a) R e_{n}=10 \& x_{o}=2 \mathrm{~mm}$, (b) $R_{n}=50 \& x_{o}$ $=2 \mathrm{~mm},(\mathrm{c}) \operatorname{Re}_{n}=10 \& x_{o}=4 \mathrm{~mm}$, and (d) $R e_{n}=50 \& x_{o}=4 \mathrm{~mm}$

Increasing the radius of curvature whilst keeping the fluid properties and velocity constant decreases the centripetal force, whilst the inertial and viscous effects remain constant. Resultantly, the conditions that previously corresponded to the secondary flow region $\left(\mathrm{De}_{\mathrm{o}}=70-200\right)$ move into the region in 
which no flow instabilities are expected $\left(\mathrm{De}_{\mathrm{o}}<70\right)$, whilst the conditions that previously corresponded to more chaotic flow, where increased axial/radial mixing occurred $\left(\mathrm{De}_{\mathrm{o}}>200\right)$, move into the secondary flow region. At the low flow rates investigated in this study, the fluid's inertia was dominated by the oscillatory velocity component (because $\operatorname{Re}_{\mathrm{o}} / \mathrm{Re}_{\mathrm{n}}>1$ ). This means increasing the amplitude provided greater inertia, which compensated for the loss of the centripetal force, resulting in the optimal amplitude increasing with larger radii of curvature. In Figure 11, the optimal amplitudes of $2 \mathrm{~mm}$ and $4 \mathrm{~mm}$ for $R_{c}=12.5 \mathrm{~mm}$ and $R_{c}=32.5 \mathrm{~mm}$ correspond to Strouhal numbers (equation 16 ) of $\mathrm{St}=0.99$ and $\mathrm{St}=1.29$ respectively. It is probably the case that the actual optimal amplitudes are not integer values, explaining the small difference in St. This could be reconciled in future studies by studying a wider range of $\mathrm{St}$ for a particular tube diameter.

There was no significant difference between the maximum number of equivalent tanks in series produced by the designs with different radii of curvature; only the oscillation conditions to achieve the maximum plug flow quality changed. This is unlike coiled flow inverters where the reduced curvature increases the plug flow enhancement [9], though the flow inverters in this study covered a much larger Reynolds number range $\left(\operatorname{Re}_{\mathrm{n}}=2000-10,000\right)$. This suggests that oscillatory motion can be used to replicate the same mixing enhancements for coiled tubes with different dimensions for various applications, providing the oscillation conditions are optimised for the specific geometry.

\subsection{Collated Results}

In figure 9, it was shown that the optimal plug flow behaviour occurred when the ratio of the oscillatory Dean number to net flow Reynolds number was between 2 and 8 . Then, it was shown that the data could be collapsed (for $x_{0} \geq 2 \mathrm{~mm}$ ) when plotting the ratio of the number of tanks-in-series to the Strouhal number $(\mathrm{N} / \mathrm{St})$. This ratio normalises the plug flow behaviour according to the relative axial and radial mixing intensities. To summarise the data collected for all $5 \mathrm{~mm}$ diameter reactor geometries therefore, the number of equivalent tanks-in-series, $\mathrm{N}$, was plotted against the modified velocity ratio $\left(\mathrm{De}_{\mathrm{o}} / \mathrm{Re}_{\mathrm{n}}\right)$ and Strouhal number (St), as shown in Figure 12a. Here, equation 18 was fitted to the data, producing an $\mathrm{R}^{2}$ value of $68 \%$. This was a result of a large spread in the experimental data around the optimum point, which could again be a consequence of the sensitivity of the tanks-in-series model at high values of N. Nonetheless, equation 18 was able to reasonably replicate the shape produced by the data (shown in Figure 12a), meaning it describes the optimal values of $\mathrm{De}_{\mathrm{o}} / \mathrm{Re}_{\mathrm{n}}$ and $\mathrm{St}$ with minimal error.

(a)

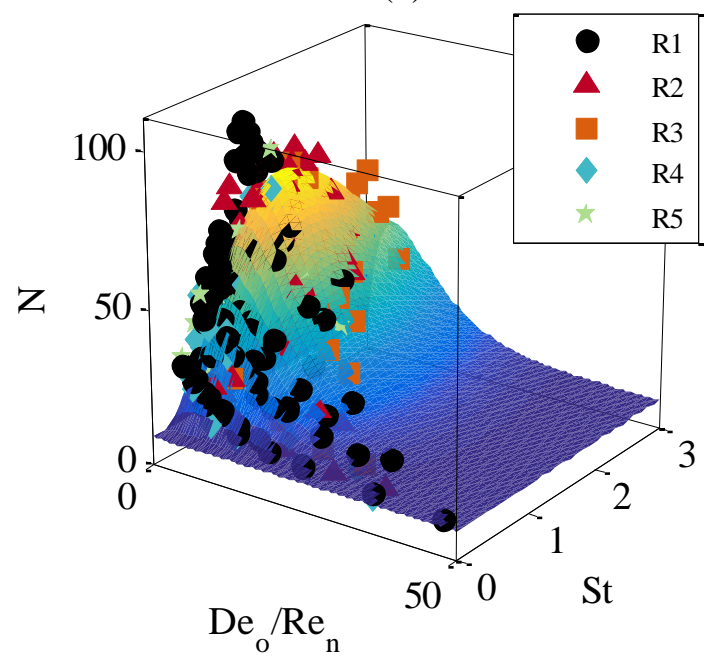

(b)

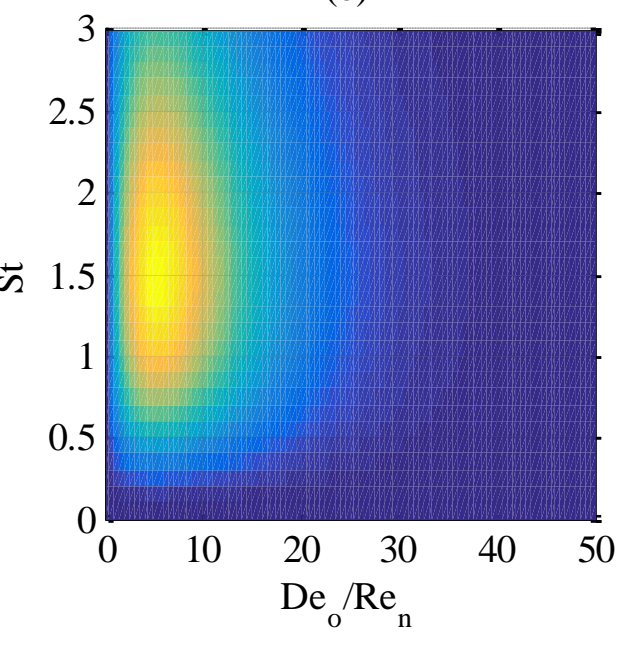

Figure 12. (a) Relationship between plug flow quality ( $N$ ) and modified velocity ratio $\left(\psi^{\prime}=D e_{d} / R e_{n}\right)$ and Strouhal number $(S t)$ at oscillation amplitudes of $x_{o} \geq 2 \mathrm{~mm}$ for all reactor geometries (RI-R5)|

(b) surface plot showing the optimal values of Deo/Re ${ }_{n}$ and St for plug flow

$$
N=9.3+142.4 S t^{1.98} \psi^{\prime 0.95} e^{-1.38 S t-0.19 \psi^{\prime}}
$$


It can be seen in Figure $12 b$ that the optimal plug flow conditions are $\mathrm{De}_{\mathrm{o}} / \mathrm{Re}_{\mathrm{n}}=2-8$ and $\mathrm{St}=1-2$ for all $5 \mathrm{~mm}$ diameter designs studied. These velocity ratios are similar to those reported for $5 \mathrm{~mm}$ diameter meso-OBRs containing central axial and integral baffles ( $\psi=4-8$ and $\psi=5-10$ respectively) [31]. There does not appear to be any significant interaction between these dimensionless groups because there is no saddle point in the plot; i.e. the optimal $\mathrm{St}$ is the same at all optimal $\mathrm{De} / \mathrm{Re}_{\mathrm{n}}$, and vice versa. The black regions in Figure $12 \mathrm{~b}$ correspond to $\mathrm{N} \approx 10$, the minimum acceptable plug flow quality, meaning these oscillatory conditions do not provide any significant enhancement compared to the steady flow only results.

\section{Conclusions}

It has been shown in this study that plug flow can be enhanced in coiled tube reactors at low flow rates $\left(\operatorname{Re}_{\mathrm{n}}=10-50\right)$ using a superimposed oscillatory fluid motion. It is likely that Dean vortices are periodically formed and unravelled in the coils in a similar manner to the toroidal vortices formed in typical oscillatory baffled reactors, preventing the formation of stagnant regions at the centres of the Dean vortices. Unlike the coiled flow inverter, where it is necessary to maintain a minimum number of turns of the coil between each inversion point, the oscillatory motion provides an enhancement of the radial mixing by disrupting the Dean vortices in time rather than in space. This decouples the mixing intensity from the reactor length, potentially allowing shorter reactors to be used for the same mean residence time process, and allowing greater freedom on the reactor design. Furthermore, the flow rates where the plug flow enhancement were observed in this study were at least as low as $\operatorname{Re}_{\mathrm{n}}=10$; further study is warranted to ascertain the true lower operating limit. These are lower than the flow rates previously reported for helical coil reactors $\left(\operatorname{Re}_{\mathrm{n}} \sim 70-300\right)$ and CFIs $\left(\operatorname{Re}_{\mathrm{n}}=15-24\right)$ but with better plug flow quality, opening up the possibility of using helical coil reactors in a larger range of processes and potentially new applications. Alternatively, it suggests that smaller (more compact) reactors could be used to generate the same mean residence times, which would minimise active material inventory.

The plug flow enhancement appeared to 'switch-on' then 'switch-off' as the oscillation amplitude and frequency were increased. These transitions correspond to the oscillatory Dean number range of $\mathrm{De}_{\mathrm{o}}=$ 70-200 for all reactor geometries. This correlates with the "secondary instability region" observed in circular geometries in steady flow, where Dean vortices are expected to form.

The tube pitch was shown to have no effect on the plug flow behaviour. This was expected because the tube pitch is not contained within the Dean number. Increasing the radius of curvature decreased the centripetal force, necessitating a higher oscillation amplitude to compensate the fluid's lost angular momentum to achieve the same plug flow enhancement. Increasing the net flow rate also required an increase of the oscillation intensity in order to compensate for a loss of radial mixing on the backstroke of the oscillation cycle. The optimal operating conditions where the maximum plug flow quality was observed were subsequently determined to be $\mathrm{De}_{\mathrm{o}} / \mathrm{Re}_{\mathrm{n}}=2-8$ and $\mathrm{St}=1-2$. The highest equivalent number of tanks-in-series observed for all designs was $\mathrm{N}=100$, which is significantly higher than the similar meso-OBR platform.

Overall, the oscillatory flow helical tube reactor is a novel form of compact plug flow reactor that can be used for flow chemistry at laboratory scales, and perhaps at larger scales, should the fluid mechanics be scalable. Through additive manufacturing, simple coiled tubes can be tailored towards targeted processes, perhaps delivering new applications by enabling the manufacture of bespoke products such as those found in the fine chemicals and pharmaceuticals industries. The use of oscillatory flow also provides a retrofitting upgrade option for established processes that use helical coil geometries with steady flows, and the simpler geometry in comparison to the coiled flow inverter may allow for rapid heat transfer arrangements or more space-efficient heat exchanger designs. 


\section{Nomenclature}

C Concentration of the tracer (mol/L)

$\mathrm{D}_{\mathrm{f}} \quad$ Diffusion coefficient/diffusivity $\left(\mathrm{m}^{2} / \mathrm{s}\right)$

$\mathrm{D}_{\mathrm{t}} \quad$ Tube diameter $(\mathrm{m})$

f Oscillation frequency $(\mathrm{Hz})$

$\mathrm{L} \quad$ Characteristic length $(\mathrm{m})$

$\mathrm{L}_{\mathrm{c}} \quad$ Length of the coiled part of the tube (m)

$\mathrm{L}_{\mathrm{T}} \quad$ Total length of the 3D printed reactor (excluding the conductivity fittings) (m)

$\mathrm{N} \quad$ Number of equivalent tanks in series

$\mathrm{P} \quad$ Pitch of the tube (rise height during one rotation of the tube) (m)

$\mathrm{R}_{\mathrm{c}} \quad$ Radius of curvature (defined using the inner part of the tube) (m)

$\mathrm{t} \quad$ Time (s)

$\mathrm{T} \quad$ Number of turns of the helical coil

U Characteristic velocity $(\mathrm{m} / \mathrm{s})$

$\mathrm{U}_{\text {osc, } \mathrm{m}}$ Maximum oscillatory velocity $(\mathrm{m} / \mathrm{s})$

$\mathrm{v} \quad$ Steady flow velocity $(\mathrm{m} / \mathrm{s})$

$\mathrm{V} \quad$ Reactor volume $(\mathrm{mL})$

$\mathrm{x}_{\mathrm{o}} \quad$ Oscillation amplitude $(\mathrm{m})$

Dimensionless Groups

De Dean number $\left(=R e_{n} \sqrt{D_{t} / 2 R_{c}}\right)$

$\mathrm{De}_{\mathrm{o}} \quad$ Oscillatory Dean number $\left(=R e_{o} \sqrt{D_{t} / 2 R_{c}}\right)$

Pe Péclet number $\left(=U L / D_{f}\right)$

$\operatorname{Re}_{\mathrm{n}} \quad$ Net Reynolds Number (also referred to as Re in the abstract/introduction) $(=\rho v d / \mu)$

$\operatorname{Re}_{o} \quad$ Oscillatory Reynolds Number $\left(=2 \pi f x_{o} \rho v / \mu\right)$

St $\quad$ Strouhal Number $\left(=f L / U=R_{c} / 2 \pi x_{o}\right)$

$\alpha \quad$ Dimensionless frequency $\left(=R_{c}(\omega / \nu)^{0.5}\right)$

$\beta \quad$ Pulsation strength $\left(=U_{o s c, m} / v\right)$

$\psi \quad$ Velocity Ratio $\left(=R e_{o} / R e_{n}\right)$

$\psi \quad$ Modified velocity ratio $\left(=D e_{o} / R e_{n}\right)$

Greek Letters

$\theta \quad$ Dimensionless time $(=t / \tau)$

$\mu \quad$ Viscosity (Pa.s)

$v \quad$ Kinematic viscosity $\left(\mathrm{m}^{2} / \mathrm{s}\right)(=\mu / \rho)$

$\rho \quad$ Density $\left(\mathrm{kg} / \mathrm{m}^{3}\right)$

$\sigma^{2} \quad$ Variance of RTD

$\tau \quad$ Mean residence time (s)

$\omega \quad$ Angular frequency $(\mathrm{Hz})(=2 \pi f)$ 


\section{References}

[1] Barua, S.N. On secondary flow in stationary curved pipes. The Quarterly Journal of Mechnaics and Applied Mathematics 16(1) (1963) 61-77

[2] Dean, W.R. Note on the Motion of Fluid in a Curved Pipe. The London, Edinburgh and Dublin Philosophical Magazine and Journal of Science 4(20) (1927) 208-223

[3] Nivedita, N., Ligrani, P. \& Papautsky, I. Dean Flow Dynamics in Low-Aspect Ratio Spiral Microchannels. Scientific Reports 7 (2017) 1-10

[4] Ligrani, P.M. A Study of Dean Vortex Development and Structure in a Curved Rectangular Channel With Aspect Ratio of 40 at Dean Numbers up to 430. US Army Research Laboratory (ARL-CR-144) and Lewis Research Centre (4607)

[5] Saxena, A.K. \& Nigam, K.D.P. Coiled Configuration for Flow Inversion and Its Effect on Residence Time Distribution. American Institute of Chemical Engineers Journal 30(3) (1984) 363-368

[6] Lyne, W.H. Unsteady viscous flow in a curved pipe. J. Fluid Mech. 45(1) (1970) 13-31

[7] Koutsky, J.A. \& Adler, R.J. Minimisation of Axial Dispersion by Use of Secondary Flow in Helical Tubes. The Canadian Journal of Chemical Engineering 42 (1964) 239-246

[8] Trivedi, R.N. \& Vasudeva, K. Axial dispersion in laminar flow in helical coils. Chemical Engineering Science 30 (1975) 317-325

[9] Sharma, L., Nigam, K.D.P. \& Roy, S. Single Phase Mixing in Coiled Tubes and Coiled Flow Inverters in Different Flow Regimes. Chemical Engineering Science 160 (2017) 227-235

[10] Mridha, M. \& Nigam, K.D.P. Coiled flow inverter as an inline mixer. Chemical Engineering Science 63 (2008) 1724-1732

[11] Sharma, A.K., Agarwal, H., Pathak, M., Nigam, K.D.P., Rathore, A.S. Continuous refolding of a biotech therapeutic in a novel Coiled Flow Inverter Reactor. Chemical Engineering Science 140 (2016) 153-160

[12] Parida, D., Serra, C.A., Garg, D.K., Hoarau, Y., Bally, F., Muller, R., Bouquey, M. Coil Flow Inversion as a Route to Control Polymerization in Microreactors. Macromoleculaes 47(10) (2014) 3282-3287

[13] Gürsel, I.V., Kurt, S.K., Aalders, J., Wang, Q., Noël, T., Nigam, K.D.P., Kockmann, N., Hessel, V. Utilization of milli-scale coiled flow inverter in combination with phase separator for continuous flow liquid-liquid extraction processes. Chemical Engineering Journal 282 (2016) 855-868

[14] Kateja, N. \& Rathore, A. Spiralling into Affordable Healthcare. The Chemical Engineer (2018) 33-35

[15] Rossi, D., Gargiulo, L., Valitov, G., Gavriilidis, A., Mazzei, L. Experimental characterization of axial dispersion in coiled flow inverters. Chemical Engineering Research \& Design 120 (2017) 159-170

[16] Singh, J. \& Nigam, K.D.P. Pilot plant study for effective heat transfer area of coiled flow inverter. Chemical Engineering and Processing: Process Intensification 102 (2016) 219-228 
[17] Kurt, S.K., Gelhausen, M.G., Kockmann, N. Axial dispersion and heat transfer in a milli/microstructured coiled flow inverter for narrow residence time distribution at laminar flow. Chemical Engineering \& Technology 38(7) (2015) 1122-1130

[18] Vashisth, S. \& Nigam, K.D.P. Experimental investigation of pressure drop during two-phase flow in a coiled flow inverter. Ind. Eng. Chem. Res. 46 (2007) 5043-5050

[19] Jarrahi, M., Castelain, C.; Peerhossaini, H. Secondary flow patterns in laminar pulsating flow through a curved pipe. Exp Fluids 50 (2011) 1539-1558

[20] Sudo, K., Sumida, M., Yamane, R. Secondary motion of fully developed oscillatory flow in a curved pipe. J. Fluid Mech. 237 (1992) 189-208

[21] Timité, B., Jarrahi, M., Castelain, C. \& Peerhossaini, H. Pulsating Flow for Mixing Intensification in a Twisted Curved Pipe. Journal of Fluids Engineering 131(12) (2009) 121104

[22] Jarrahi, M., Castelain, C., Peerhossaini, H. Mixing enhancement by pulsating chaotic advection. Chemical Engineering and Processing: Process Intensification 74 (2013) 1-13

[23] Law, R. Ahmed, S.M.R., Tang, N., Phan, A.N. \& Harvey, A.P. Development of a more robust correlation for predicting heat transfer performance in oscillatory baffled reactors. Chemical Engineering and Processing: Process Intensification 125 (2018) 133-138

[24] Ikwebe, J. Intensification of bioethanol production by simultaneous saccharification and ferm3entation in an oscillatory baffled reactor [PhD Thesis]. Newcastle University, Newcastle upon Tyne, 2013

[25] McGlone, T., Briggs, N.E.B., Clark, C.A., Brown, C.J., Sefcik, J. \& Florence, A.J. Oscillatory Flow Reactors (OFRs) for continuous manufacturing and crystallization. Org. Process Res. Dev. 19(9) (2015) 1186-1202

[26] Masngut, N. Harvey, A.P., Ikwebe, J. Potential uses of oscillatory based reactors for biofuel production. Biofuels 1(4) (2010) 605-619

[27] Al-Abduly, A., Christensen, P. Harvey, A. \& Zhang, K. Characterization and optimization of an oscillatory baffled reactor (OBR) for ozone-water mass transfer. Chemical Engineering and Processing: process Intensification 84 (2014) 82-89

[28] Ni, X., Zhang, Y. \& Mustafa, I. Correlation of polymer particle size with droplet size in suspension polymerisation of methylmethacrylate in a batch oscillatory-baffled reactor. Chemical Engineering Science 54 (1999) 841-850

[29] Koh, M.Y., Mohd. Ghazi, T.I., Idris, A. Synthesis of palm based biolubricant in an oscillatory flow reactor (OFR). Industrial Crops and Products 52 (2014) 567-574

[30] McDonough, J.R. Phan, A.N., Harvey, A.P. Rapid process development using oscillatory baffled mesoreactors - A state-of-the-art review. Chemical Engineering Journal 265 (2015) $110-121$

[31] Phan, A.N. \& Harvey, A. Development and evaluation of novel designs of continuous mesoscale. Chemical Engineering Journal 159 (2010) 212-219

[32] Phan, A.N. \& Harvey, A.P. Characterisation of Mesoscale Oscillatory Helical Baffled Reactor - Experimental Approach. Chemical Engineering Journal 180 (2012) 229-236 
[33] MacDonald, N.P., Cabot, J.M., Smejkal, P., Guijt, R.M., Paull, B. \& Breadmore, M.C. Comparing Microfluidic Performance of Three-Dimensional (3D) Printing Platforms. Anal. Chem. 89 (2017) 3858-3866

[34] Okafor, O., Weilhard, A., Fernandes, J.A., Karjalainen, E., Goodridge, R. \& Sans, V. Advanced reactor engineering with 3D printing for the continuous-flow synthesis of solver nanoparticles. React. Chem. Eng. 2 (2017) 129-136

[35] McDonough, J.R., Ahmed, S.M.R., Phan, A.N., Harvey, A.P. A study of the flow structures generated by oscillating flows in a helical baffled tube. Chemical Engineering Science 171 (2017) 160-178

[36] Phan, A.N., Harvey, A. \& Lavender, J. Characterisation of fluid mixing in novel designs of mesoscale oscillatory baffled reactors operating at low flow rates $(0.3-0.6 \mathrm{~mL} / \mathrm{min})$. Chemical Engineering and Processing: Process intensification 50 (2011) 254-263

[37] Levenspiel, O., Chemical Reaction Engineering (3rd Edition). John Wiley and Sons, 1999

[38] Ni, X., Mackley, M.R., Harvey, A.P., Stonestreet, P., Baird, M.H.I. \& Rama Rao, N.V. Mixing Through Oscillations and Pulsations - A Guide to Achieving Process Enhancements in the Chemical and Process Industries. Chemical Engineering Research and Design 81(3) (2003) 373-383

[39] Reis, N., Harvey, A.P., Mackley, M.R., Vicente, A.A. \& Teixeira, J.A. Fluid mechanics and design aspects of a novel oscillatory flow screening mesoreactor. Chemical Engineering Research and Design 83(A4) (2005) 357-371

[40] Phan, A.N., Harvey, A.P. Effect of geometrical parameters on fluid mixing in novel mesoscale oscillatory helical baffled designs. Chemical Engineering Journal 169 (2011) 339347

[41] Nogueira, X., Taylor, B.J., Gomez, H., Colominas, I. \& Mackley, M.R. Experimental and computational modelling of oscillatory flow within a baffled tube containing periodic-triorifice baffle geometries. Computers and Chemical Engineering 49 (2013) 1-17

[42] Klutz, S.; Kurt, S.K., Lobedann, M., Kockmann, N. Narrow residence time distribution in tubular reactor concept for Reynolds number range of 10-100. Chemical Engineering Research and Design 95 (2015) 22-33

[43] Fogler, H.S. (2016) Elements of Chemical Reaction Engineering [5th ed.]. Upper Saddle River, N.J. :Prentice Hall PTR

[44] Stonestreet, P. \& Van Der Veeken, P.M.J. The effects of oscillatory flow and bulk flow components on residence time distribution in baffled tube reactors. Trans IChemE 77(A) (1999) 671-684

[45] Tang, L., Tang, Y., Parameswaran, S. A numerical study of flow characteristics in a helical pipe. Advances in Mechanical Engineering 8(7) (2016) 1-8

[46] Ali, S. Pressure Drop Correlations for Flow Through Regular. Fluid Dynamics Research 28 (2001) 295-310

[47] Kumar, V., Aggarwal, M. \& Nigam, K.D.P. Mixing in Curved Tubes. Chemical Engineering Science 61 (2006) 5742-5753 